\title{
Existence of solutions with a horizon in pure scalar-Gauss-Bonnet theories
}

\author{
A. Bakopoulos@,$^{1, *}$ P. Kanti®, ${ }^{1, \dagger}$ and N. Pappas $\oplus^{1,2, \hbar}$ \\ ${ }^{1}$ Division of Theoretical Physics, Department of Physics, University of Ioannina, \\ Ioannina GR-45110, Greece \\ ${ }^{2}$ Nuclear and Particle Physics Section, Physics Department, National and Kapodistrian University of Athens, \\ Athens GR-15771, Greece
}

(Received 6 November 2019; accepted 24 January 2020; published 13 February 2020)

\begin{abstract}
We consider the Einstein-scalar-Gauss-Bonnet theory and assume that, at regimes of large curvature, the Ricci scalar may be ignored compared to the quadratic Gauss-Bonnet term. We then look for static, spherically symmetric, regular black-hole solutions with a nontrivial scalar field. Despite the use of a general form of the spacetime line element, no black-hole solutions are found. In contrast, solutions that resemble irregular particlelike solutions or completely regular gravitational solutions with a finite energymomentum tensor do emerge. In addition, in the presence of a cosmological constant, solutions with a horizon also emerge, however, the latter corresponds to a cosmological rather than to a black-hole horizon. It is found that, whereas the Ricci term works towards the formation of the positively curved topology of a black-hole horizon, the Gauss-Bonnet term exerts a repulsive force that hinders the formation of the black hole. Therefore, a pure scalar-Gauss-Bonnet theory cannot sustain any black-hole solutions. However, it could give rise to interesting cosmological or particlelike solutions where the Ricci scalar plays a less fundamental role.
\end{abstract}

DOI: 10.1103/PhysRevD.101.044026

\section{INTRODUCTION}

After a century of searching and anticipation, at last, gravitational waves - signals from processes taking place in strong-gravity regimes in our Universe-have been successfully detected $[1,2]$. This development has refueled the interest in the construction of a more fundamental theory of gravity. In most cases, such a theory includes extra fields or higher-curvature terms in its action [3,4], the presence of which modifies the characteristics of the emergent gravitational solutions compared to the ones arising in the context of the traditional general relativity (GR).

The quest for novel black-hole solutions in the context of a generalized gravitational theory has been the most intense of all. The restrictive no-hair theorem [5] of GR, which applied to gravitational theories including minimally coupled scalar fields, was evaded when novel black-hole solutions with Yang-Mills [6], Skyrme fields [7] or fields with a conformal coupling to gravity [8] appeared in the literature. A novel formulation of the no-hair theorem [9]

\footnotetext{
*a.bakop@uoi.gr

pkanti@uoi.gr

"npappas@uoi.gr
}

Published by the American Physical Society under the terms of the Creative Commons Attribution 4.0 International license. Further distribution of this work must maintain attribution to the author(s) and the published article's title, journal citation, and DOI. Funded by SCOAP. was also evaded in the context of a gravitational theory with a scalar field coupled to the Gauss-Bonnet term, a quadratic curvature term [10-12]: the discovery of the dilatonic black holes [13] (see also [14-18] for some earlier studies) was soon followed by the one of the colored black holes [19,20] in the presence of a Yang-Mills field, and then of higherdimensional [21] or rotating versions [22-25] (see [26-29] for a number of reviews).

After a dormant period, the revival of the Horndeski [30] and Galileon [31] theories gave a significant boost to the concept of generalized gravitational theories, which contain a single scalar field and higher-derivative curvature terms. Even the no-hair theorems were re-formulated $[32,33]$ but to no avail: novel black-hole solutions were again constructed [34-37]. These solutions, as well as the earlier ones mentioned above, have the characteristic feature of the scalar hair: a regular, nontrivial scalar field that is associated with the black hole, a feature forbidden by GR. In a recent work [38], it was demonstrated that a general class of theories with a scalar field having an arbitrary coupling function to the quadratic Gauss-Bonnet term, the Einsteinscalar-Gauss-Bonnet class of theories, always evades the no-hair theorem [9] and leads to regular, black-hole solutions with scalar hair. The early dilatonic [13] and shift-symmetric Galileon [36] solutions are particular examples of this general statement - as are also the additional solutions $[39,40]$ that appeared almost simultaneously with [38]. In addition, a large number of works has 
appeared that studied novel black holes or compact objects in these, or similar, types of theories as well as their properties [41-115]. The asymptotically flat black-hole solutions were also supplemented by solutions with an asymptotic (anti)-de Sitter behavior, a topic that has also attracted a lot of interest in the literature [116-134].

The Einstein-scalar-Gauss-Bonnet theory has in fact proven to be an extremely rich generalized theory of gravity. Apart from novel black-hole solutions as described above, it has been shown to lead to families of wormholes that require no exotic matter $[135,136]$ and particlelike solutions with regular spacetimes [130,137], all with nontrivial scalar hair (see also [138-142]). The presence of the quadratic Gauss-Bonnet term seems to be of paramount importance for the emergence of all of these compact solutions. It creates an effective energy-momentum tensor that may locally violate the energy conditions while the actual matter fields of the theory continue to respect them. This leads to the evasion of nonexistence arguments of GR and the emergence of novel solutions, from black holes to wormholes, with a scalar hair.

The Einstein-scalar-Gauss-Bonnet theory has also interesting cosmological implications, and these were in fact the first ones to be studied in the literature. In the context of the effective heterotic superstring theory, where the scalar field was identified with one of the moduli fields of the theory, it was shown that this theory leads to singularity-free cosmological solutions [143]. Later, it was demonstrated that a similar type of solutions emerges for a variety of coupling functions, with a number of common features, between the scalar field and the Gauss-Bonnet term [144,145]. More recently, the same theory was studied from a novel perspective [146]: as we go backwards in time, the curvature of spacetime considerably increases and the quadratic Gauss-Bonnet term becomes eventually as important as, or even larger than, the linear Ricci term. Assuming that such a time period exists, the Ricci term was altogether ignored from the theory and the coupled system of the scalar field and the Gauss-Bonnet was studied on its own. It was found [146] that this simplified theory supported singularity-free solutions with the same characteristics as the ones emerging in the context of the complete theory. In addition, a family of attractive inflationary solutions with a natural exit mechanism was found, all in an analytical way due to the simplification of the set of field equations.

In the context of the present work, we will keep the same perspective but focus on the emergence of solutions with a horizon. Our main priority will be to investigate whether the simplified, pure scalar-Gauss-Bonnet theory leads to regular black-hole solutions with a nontrivial scalar field. We will therefore assume that there exists a part of spacetime where the Gauss-Bonnet term may dominate over the linear Ricci term. We will seek for static, spherically symmetric, regular black holes, and attempt to solve the simplified set of field equations by using both analytical and numerical methods. We will also use alternative forms of line elements in an effort to increase the flexibility of our ansatz. The presence of a cosmological constant will also be employed, and its role to the formation of a horizon will be investigated. As we will demonstrate, the pure scalarGauss-Bonnet theory cannot support by itself black-hole spacetimes - this will be due to the conflicting roles of the Gauss-Bonnet and Ricci terms in the formation of a regular black-hole horizon. Nevertheless, our quest for black-hole solutions will lead us instead to a number of alternative solutions-all parts of the phase space of solutions of the pure scalar-Gauss-Bonnet theory-with a number of interesting characteristics.

The outline of the present work is as follows: in Sec. II, we present our theoretical framework and field equations. In Sec. III, we look, in an analytical way, for solutions with a horizon in the case where the cosmological constant vanishes-we also perform an exact numerical study of a family of regular solutions we derive. In Sec. IV, we employ a generalized form of the spacetime line element, and repeat our previous analysis. We reinstate the cosmological constant in Sec. V, and interpret the solutions we obtain. In Sec. VI, we use exact numerical results for blackhole solutions found in the context of the complete Einstein-scalar-Gauss-Bonnet theory, and investigate the role of the Gauss-Bonnet and Ricci terms in the formation of a black-hole horizon. We finish with our conclusions in Sec. VII.

\section{THE THEORETICAL FRAMEWORK}

The starting point of our analysis will be the following action, describing a generalized theory of gravity,

$S=\frac{1}{16 \pi} \int d^{4} x \sqrt{-g}\left[R-\frac{1}{2} \partial_{\mu} \phi \partial^{\mu} \phi+f(\phi) R_{\mathrm{GB}}^{2}-2 \Lambda\right]$.

The theory contains the Ricci scalar curvature $R$, a scalar field $\phi$ and the higher-curvature, quadratic Gauss-Bonnet (GB) term defined as

$$
R_{\mathrm{GB}}^{2}=R_{\mu \nu \rho \sigma} R^{\mu \nu \rho \sigma}-4 R_{\mu \nu} R^{\mu \nu}+R^{2},
$$

in terms of the Riemann tensor $R_{\mu \nu \rho \sigma}$, Ricci tensor $R_{\mu \nu}$ and Ricci scalar $R$. The GB term, being a topological invariant in four dimensions, must be coupled to the scalar field $\phi$. This is realized via the arbitrary coupling function $f(\phi)$; choosing different forms for the coupling function, one may study the emergence of solutions within a whole class of theories. The theory includes also a cosmological constant $\Lambda$. Throughout our work, we will use units in which $G=c=1$.

Taking the variation of the action with respect to the metric $g_{\mu \nu}$ and the scalar field $\phi$, we end up with the gravitational field equations and the equation of motion for the scalar field. These have the following forms: 


$$
\begin{aligned}
G_{\mu \nu} & =T_{\mu \nu}, \\
\nabla^{2} \phi+\dot{f}(\phi) R_{\mathrm{GB}}^{2} & =0,
\end{aligned}
$$

respectively. In the above, $G_{\mu \nu}$ is the Einstein tensor and $T_{\mu \nu}$ is the total energy-momentum tensor of the theory,

$$
\begin{aligned}
T_{\mu \nu}= & -\frac{1}{4} g_{\mu \nu} \partial_{\rho} \phi \partial^{\rho} \phi+\frac{1}{2} \partial_{\mu} \phi \partial_{\nu} \phi \\
& -\frac{1}{2}\left(g_{\rho \mu} g_{\lambda \nu}+g_{\lambda \mu} g_{\rho \nu}\right) \eta^{\kappa \lambda \alpha \beta} \tilde{R}_{\alpha \beta}^{\rho \gamma} \nabla_{\gamma} \partial_{\kappa} f(\phi)-\Lambda g_{\mu \nu},
\end{aligned}
$$

which receives contributions from the kinetic term of the scalar field, the GB term and the cosmological constant. The dot over the coupling function denotes its derivative with respect to the scalar field (i.e., $\dot{f}=d f / d \phi$ ). We have also used the definition

$$
\tilde{R}_{\alpha \beta}^{\rho \gamma} \equiv \eta^{\rho \gamma \sigma \tau} R_{\sigma \tau \alpha \beta} \equiv \frac{\epsilon^{\rho \gamma \sigma \tau}}{\sqrt{-g}} R_{\sigma \tau \alpha \beta} .
$$

The emergence of regular black-hole solutions with a nontrivial scalar field, in the context of the theory (1) and for a variety of coupling functions $f(\phi)$, was demonstrated in $[38,131]$ with either Minkowski or anti-de Sitter asymptotic behavior. Here, we will investigate whether regular, black-hole solutions with scalar hair emerge in the context of the pure scalar-GB theory, i.e., in the absence of the Ricci scalar from the theory. In that case, the derived solutions would rely solely on the synergy between the scalar field and the GB term. From the field equations (3) and (4), we may see that such a synergy is in principle possible: a nontrivial scalar field ensures the presence of the GB term in the theory whereas the GB term provides in its turn a nontrivial potential for the scalar field. It is this same synergy that leads to singularity-free or inflationary cosmological solutions, even in the absence of the Ricci scalar from the theory, as was analytically demonstrated in [146].

The assumption that the linear gravitational Ricci term may be ignored from the theory when compared to the quadratic GB term may be justified only in regimes of spacetime where the curvature is particularly large. This may be realized only near the black-hole horizon whereas in the asymptotic regime the Ricci scalar must be necessarily reinstated. Therefore, the question we would like to pose, and investigate in what follows, is the following: does the curvature of spacetime ever become so strong that a black-hole horizon may be formed only due to the effect of the GB term (supplemented by that of the scalar field)?

To this end, we will assume a static, spherically symmetric ansatz for the spacetime line element of the form:

$$
d s^{2}=-e^{A(r)} d t^{2}+e^{B(r)} d r^{2}+r^{2}\left(d \theta^{2}+\sin ^{2} \theta d \varphi^{2}\right) .
$$

In accordance to the above discussion, we will focus on the "near" regime of spacetime, i.e., on the small- $r$ regime, and assume that, there, all terms associated with the Ricci term may be ignored from the field equations. That amounts to ignoring altogether the components of the Einstein tensor $G_{\mu \nu}$ from the gravitational field equations (3). Then, employing the ansatz (1), the explicit form of the components of Einstein's equations becomes

$$
\begin{aligned}
T^{t}{ }_{t}= & -\frac{e^{-2 B}}{4 r^{2}}\left[\phi^{\prime 2}\left(r^{2} e^{B}+16 \ddot{f}\left(e^{B}-1\right)\right)\right. \\
& \left.-8 \dot{f}\left(B^{\prime} \phi^{\prime}\left(e^{B}-3\right)-2 \phi^{\prime \prime}\left(e^{B}-1\right)\right)\right]-\Lambda=0, \\
T^{r}= & \frac{e^{-B} \phi^{\prime}}{4}\left[\phi^{\prime}-\frac{8 e^{-B}\left(e^{B}-3\right) \dot{f} A^{\prime}}{r^{2}}\right]-\Lambda=0, \\
T^{\theta}{ }_{\theta}= & T^{\varphi}{ }_{\varphi}=-\frac{e^{-2 B}}{4 r}\left[\phi^{\prime 2}\left(r e^{B}-8 \ddot{f} A^{\prime}\right)\right. \\
& \left.-4 \dot{f}\left(A^{\prime 2} \phi^{\prime}+2 \phi^{\prime} A^{\prime \prime}+A^{\prime}\left(2 \phi^{\prime \prime}-3 B^{\prime} \phi^{\prime}\right)\right)\right]-\Lambda=0 .
\end{aligned}
$$

We observe that, upon ignoring the components of the Einstein tensor from the field equations, the total effective energy-momentum tensor vanishes. However, this is due not to the triviality of the matter distribution in our theory but to the cancellation of the positive contribution of the kinetic term of the scalar field and the negative contribution of the GB term to the effective energy-density and pressure components of the system. We therefore look for nontrivial configurations of the scalar field and metric functions that satisfy the above equations and thus correspond to locally zero-energy and zero-pressure solutions.

The scalar field equation (4) on the other hand remains unaltered by the elimination of the Ricci scalar in the small$r$ regime, and assumes the following explicit form:

$$
\begin{aligned}
& 2 r \phi^{\prime \prime}+\left(4+r A^{\prime}-r B^{\prime}\right) \phi^{\prime} \\
& \quad+\frac{4 \dot{f} e^{-B}}{r}\left[\left(e^{B}-3\right) A^{\prime} B^{\prime}-\left(e^{B}-1\right)\left(2 A^{\prime \prime}+A^{\prime 2}\right)\right]=0,
\end{aligned}
$$

where we have assumed that the scalar field shares the symmetry of spacetime and thus is also static and spherically symmetric, $\phi=\phi(r)$. In all the above equations, the prime denotes differentiation with respect to the radial coordinate $r$.

\section{SOLUTIONS IN THE NEAR REGIME FOR $\Lambda=0$}

The issue of the emergence of regular, black-hole solutions from the system of Eqs. (8)-(11), with $\Lambda=0$, was briefly discussed in [38]. Here, we will first review and expand on the mathematical arguments involved in that analysis and, second, study and characterize the families of 
solutions we obtain. We will again assume that the cosmological constant is zero and postpone the study of its role for a later section.

As our priority is to find solutions with a black-hole horizon, we will demand that, for some value of the radial coordinate $r=r_{h}$, the following conditions should hold:

$$
\left.g_{t t}\right|_{r=r_{h}^{+}} \rightarrow 0,\left.\quad g_{r r}\right|_{r=r_{h}^{+}} \rightarrow \infty .
$$

The above conditions amount to assuming that $A^{\prime} \rightarrow \infty$ and $B^{\prime} \rightarrow-\infty$. In fact, one may consider any of the two conditions as the starting point of the analysis-in the case of the emergence of a spherically symmetric black hole, these two conditions are equivalent; however, in the absence of such a solution, each condition allows us to explore different parts of the phase space of the solutions of the theory. We investigate these two different lines of thinking in the following two subsections.

\section{A. Expanding around $\boldsymbol{A}^{\prime} \rightarrow \infty$}

Before applying any limit, we may observe that Eq. (9) can be solved to yield an expression for the metric component $e^{B}$, that is

$$
e^{B}=\frac{24 A^{\prime} \dot{f}}{8 A^{\prime} \dot{f}-r^{2} \phi^{\prime}},
$$

from which we may easily deduce an expression for the first derivative of $B$, namely

$$
B^{\prime}=\frac{r\left[A^{\prime}\left(\dot{f}\left(r \phi^{\prime \prime}+2 \phi^{\prime}\right)-r \phi^{\prime 2} \ddot{f}\right)-r A^{\prime \prime} \phi^{\prime} \dot{f}\right]}{A^{\prime} \dot{f}\left(8 A^{\prime} \dot{f}-r^{2} \phi^{\prime}\right)} .
$$

Employing Eqs. (13) and (14), we may eliminate the metric function $B(r)$ and its derivative from the remaining field equations (8), (10) and (11). The latter reduce to a set of two independent second-order, coupled, ordinary differential equations that may be brought to the following form:

$$
A^{\prime \prime}=\frac{P}{S}, \quad \phi^{\prime \prime}=\frac{Q}{S},
$$

where

$$
\begin{aligned}
S= & 16 e^{B} \dot{f}\left(16 A^{\prime} \dot{f}-r^{2} \phi^{\prime}\right)+16 \dot{f}\left(5 r^{2} \phi^{\prime}-16 A^{\prime} \dot{f}\right), \\
P= & e^{2 B}\left(32 r A^{\prime} \phi^{\prime} \dot{f}-6 r^{3} \phi^{\prime 2}\right) \\
& +e^{B}\left(-64 r^{2} A^{\prime} \phi^{\prime 2} \ddot{f}+40 r^{2} A^{\prime 2} \phi^{\prime} \dot{f}+32 r A^{\prime} \phi^{\prime} \dot{f}\right. \\
& \left.-128 A^{\prime 3} \dot{f}^{2}-5 r^{4} A^{\prime} \phi^{\prime 2}+10 r^{3} \phi^{\prime 2}\right)-40 r^{2} A^{\prime 2} \phi^{\prime} \dot{f} \\
& +128 A^{\prime 3} \dot{f}^{2},
\end{aligned}
$$

$$
\begin{aligned}
Q= & -\frac{1}{A^{\prime}}\left[e ^ { B } \phi ^ { \prime 2 } \left(16 r^{2} A^{\prime} \phi^{\prime} \ddot{f}+8 r^{2} A^{\prime 2} \dot{f}-32 r A^{\prime} \dot{f}\right.\right. \\
& \left.+256 A^{\prime 2} \dot{f} \ddot{f}+r^{4} A^{\prime} \phi^{\prime}-6 r^{3} \phi^{\prime}\right) \\
& +\phi^{\prime 2}\left(-16 r^{2} A^{\prime} \phi^{\prime} \ddot{f}+24 r^{2} A^{\prime 2} \dot{f}+96 r A^{\prime} \dot{f}-256 A^{\prime 2} \dot{f} \ddot{f}\right) \\
& \left.+2 r^{3} e^{2 B} \phi^{\prime 3}\right] .
\end{aligned}
$$

In the above expressions, we have eliminated $B^{\prime}$ but, for simplicity of notation, we kept $e^{B}$-the latter should be considered as a dependent function of the remaining independent variables according to Eq. (13).

We will now assume that, as $r \rightarrow r_{h}, A^{\prime}$ diverges. The regularity of the horizon, if existent, demands that the scalar field $\phi$ as well as its first and second derivative remain there finite. Under these assumptions, Eq. (13) readily gives that

$$
e^{B}=3+\mathcal{O}\left(\frac{1}{A^{\prime}}\right)
$$

By replacing the above in Eqs. (15)-(18), we find that, near the point of interest, the following relations hold:

$$
A^{\prime \prime}=-\frac{1}{2} A^{\prime 2}+\mathcal{O}\left(A^{\prime}\right), \quad \phi^{\prime \prime}=\mathcal{O}(1)
$$

The above equations may be easily integrated with respect to the radial coordinate to give

$A^{\prime}=\frac{2}{r-r_{h}}+\mathcal{O}(1), \quad \phi^{\prime}=\phi_{1}\left(r-r_{h}\right)+\mathcal{O}(1)$.

The first of the above equations consistently gives that, near $r_{h}, A^{\prime} \rightarrow \infty$ as assumed above. Integrating once more, we find the complete form of the asymptotic solution for the metric functions $A, B$ and the scalar field $\phi$ near $r_{h}$ :

$e^{A}=a_{2}\left(r-r_{h}\right)^{2}+\mathcal{O}\left(\left(r-r_{h}\right)^{3}\right)$,

$e^{B}=3+b_{1}\left(r-r_{h}\right)+\mathcal{O}\left(\left(r-r_{h}\right)^{2}\right)$,

$\phi=\phi_{0}+\phi_{1}\left(r-r_{h}\right)+\phi_{2}\left(r-r_{h}\right)^{2}+\mathcal{O}\left(\left(r-r_{h}\right)^{3}\right)$.

In the above, $b_{1}, a_{2}$ and $\phi_{i}$ are arbitrary integration constants. As demanded, the scalar field and its derivatives are regular at $r_{h}$ while the metric function $e^{A}$ vanishes thus exhibiting the expected behavior near a black-hole horizon. However, the behavior of the second metric function $e^{B}$, as given by Eq. (23), does not describe a black hole as it remains regular near $r_{h}$. In order to gain more information about the form of spacetime around $r_{h}$, we calculate the scalar gravitational quantities, the exact expressions of which may be found in the Appendix A. We then find the following results: 


$$
\begin{aligned}
R & =-\frac{10 b_{1}}{3\left(r-r_{h}\right)}+\mathcal{O}(1), \\
R_{\mu \nu} R^{\mu \nu} & =\frac{50 b_{1}}{9\left(r-r_{h}\right)^{2}}+\mathcal{O}\left(\frac{1}{r-r_{h}}\right), \\
R_{\mu \nu \rho \sigma} R^{\mu \nu \rho \sigma} & =\frac{100 b_{1}}{9\left(r-r_{h}\right)^{2}}+\mathcal{O}\left(\frac{1}{r-r_{h}}\right), \\
R_{\mathrm{GB}}^{2} & =-\frac{40 b_{1}}{3 r_{h}^{2}\left(r-r_{h}\right)}+\mathcal{O}(1) .
\end{aligned}
$$

According to the above, all curvature invariant quantities $R$, $R_{\mu \nu} R^{\mu \nu}$ and $R_{\mu \nu \rho \sigma} R^{\mu \nu \rho \sigma}$ are diverging near $r_{h}$ while the Gauss-Bonnet combination exhibits a softer divergence than expected as the dominant terms of order $\left(r-r_{h}\right)^{-2}$ exactly cancel.

Under the change of coordinate $l=r-r_{h}$, the expansions (24) resemble the asymptotic form of a particlelike solution near the origin. As discussed above, our solution is characterized by a spacetime singularity that, at first, may be considered as unphysical. However, particlelike solutions plagued by singularities, either in spacetime or in the profile of the scalar field, are quite common in the literature in the context of scalar-tensor theories of gravity (see, for instance [137,139]). These solutions are physical whenever they are characterized by a finite total energymomentum tensor as is the case also for the solutions derived here.

\section{B. Expanding around $\boldsymbol{B}^{\prime} \rightarrow-\infty$}

Alternatively, we may employ the fact that near a blackhole horizon it holds that $e^{B} \rightarrow \infty$. As we will shortly confirm, this amounts to assuming that $B^{\prime} \rightarrow-\infty$. The metric function $A$ will now be considered as a dependent variable, and Eq. (9) may be readily solved to give

$$
A^{\prime}=\frac{r^{2} e^{B} \phi^{\prime}}{8\left(e^{B}-3\right) \dot{f}} .
$$

Computing also the second derivative of $A$ from the above expression, we may eliminate the metric function $A$ and its derivatives from the remaining field equations. Then, we form a system of two coupled, ordinary differential equations, one first order and one second order, for the metric function $B$ and the scalar field $\phi$, respectively. These have the form

$$
B^{\prime}=\frac{\mathcal{Y}}{\mathcal{W}}, \quad \phi^{\prime \prime}=\frac{\mathcal{X}}{\mathcal{W}},
$$

where

$$
\begin{aligned}
\mathcal{W}= & 32 A^{\prime} \dot{f} \\
\mathcal{Y}= & 2 e^{-B}\left[-2\left(e^{B}-1\right)\left(r e^{B} \phi^{\prime}-8 A^{\prime \prime} \dot{f}\right)+8\left(e^{B}-1\right) A^{\prime 2} \dot{f}\right. \\
& \left.+r^{2}\left(-e^{B}\right) A^{\prime} \phi^{\prime}\right] \\
\mathcal{X}= & 2 e^{-B} \phi^{\prime}\left(e^{B}-3\right)\left(r e^{B} \phi^{\prime}-8 A^{\prime \prime} \dot{f}\right) \\
& +e^{B} A^{\prime} \phi^{\prime}\left(32 \ddot{f}+3 r^{2}\right)-8\left(e^{B}-3\right) A^{\prime 2} \dot{f}
\end{aligned}
$$

Again, for notational simplicity we have kept $A^{\prime}$ and $A^{\prime \prime}$ in the expressions above, however, these quantities are now dependent functions of the independent variables $B$ and $\phi$.

Let us now focus on the regime near $r_{h}$, where $e^{B}$ and $B^{\prime}$ are assumed to diverge. First, we expand there Eq. (29) to obtain

$$
A^{\prime}=\frac{r^{2} \phi^{\prime}}{8 \dot{f}}+\mathcal{O}\left(e^{-B}\right)
$$

Substituting the above result into Eqs. (30)-(32), we find that near $r_{h}$ the following relations hold:

$$
\begin{aligned}
B^{\prime} & =-\frac{2}{r} e^{B}+\mathcal{O}\left(e^{-B}\right), \\
\phi^{\prime \prime} & =-\frac{e^{B}}{r} \phi^{\prime}+\mathcal{O}\left(e^{-B}\right) .
\end{aligned}
$$

According to Eq. (35), near $r_{h}, B^{\prime}$ diverges indeed to minus infinity as assumed. If we integrate this equation with respect to the radial coordinate, we find that

$$
e^{-B}=2 \ln \left(\frac{r}{r_{h}}\right)
$$

Indeed, as demanded, the metric function $e^{B}$ exhibits the desired behavior near a black-hole horizon. In order for this horizon to be also regular, the scalar field and its derivatives should be finite. Then, Eq. (36) dictates that we must necessarily have $\phi^{\prime}\left(r_{h}\right)=0$. We may in fact solve analytically Eqs. (34) and (36) to obtain the solutions

$\phi=\phi_{0}+\phi_{1}\left(-\frac{1}{2} \sqrt{\pi} r_{h} \operatorname{Erfi}(\xi)+r \xi\right), \quad \phi^{\prime}=\phi_{1} \xi$,

and

$$
A=a_{0}-\frac{r^{3} \phi_{1}\left(\sqrt{3} F_{D}(\sqrt{3} \xi)-3 \xi\right)}{72 \dot{f}\left(\phi_{0}\right)}
$$

where $a_{0}$ and $\phi_{i}$ are again integration constants, and where we have defined the variable 


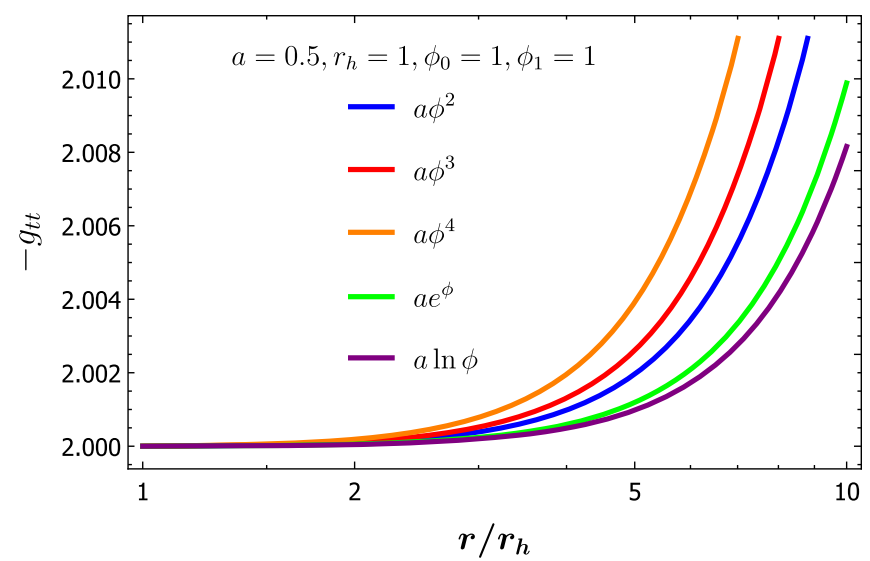

(a)

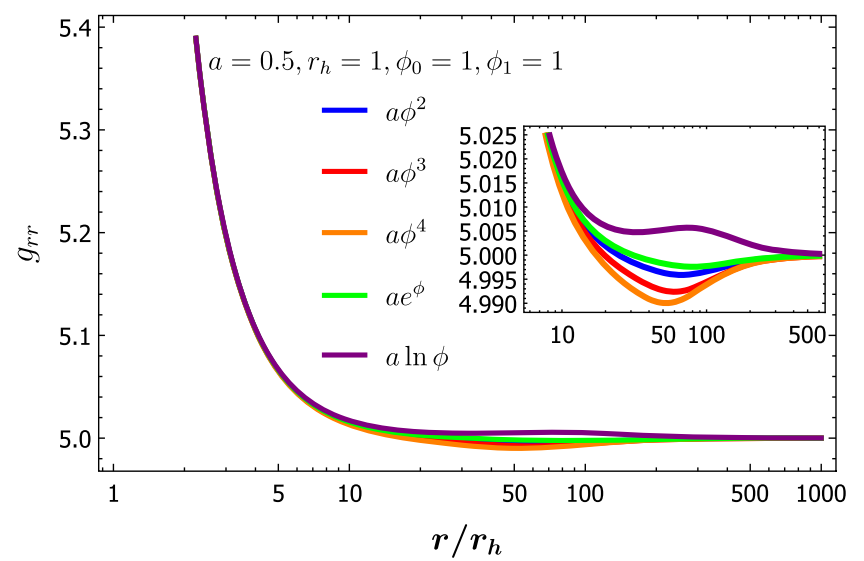

(b)

FIG. 1. (a) The $-g_{t t}$ component, and (b) the $g_{r r}$ component of the metric tensor for a pure scalar-Gauss-Bonnet solution and for a variety of forms of the coupling function $f(\phi)$.

$$
\xi=\sqrt{\ln \left(\frac{r}{r_{h}}\right)}
$$

Also, $F_{D}(x)$ and $\operatorname{Erfi}(x)$ are the Dawson and error function, respectively, defined as

$$
F_{D}(x)=e^{-x^{2}} \int_{0}^{x} e^{t^{2}} d t=\frac{\sqrt{\pi}}{2} e^{-x^{2}} \operatorname{Erfi}(x) .
$$

Although the solution for the metric function $e^{B}$ (37) hints to the presence of a horizon, on which the scalar field remains regular according to Eq. (38), the behavior of the metric function $e^{A}$ reveals that this solution is not a black hole: in the limit $r \rightarrow r_{h}$, or $\xi \rightarrow 0, A^{\prime}$ remains finite and $A$ adopts an arbitrary constant value. Using the above asymptotic solutions, we may calculate once again the scalar curvature quantities, the expressions of which are listed below:

$$
\begin{aligned}
R & =-\frac{2}{r_{h}^{2}}+\mathcal{O}\left(\sqrt{r-r_{h}}\right), \\
R_{\mu \nu} R^{\mu \nu} & =\frac{4}{r_{h}^{4}}+\mathcal{O}\left(\sqrt{r-r_{h}}\right), \\
R_{\mu \nu \rho \sigma} R^{\mu \nu \rho \sigma} & =\frac{12}{r_{h}^{4}}+\mathcal{O}\left(\sqrt{r-r_{h}}\right), \\
R_{\mathrm{GB}}^{2} & =-\frac{\phi_{1} \sqrt{r-r_{h}}}{r_{h}^{3 / 2} \dot{f}\left(\phi_{0}\right)}+\mathcal{O}\left(\left(r-r_{h}\right)^{3 / 2}\right) .
\end{aligned}
$$

The above asymptotic values reveal that, near $r_{h}$, the spacetime remains regular and no singularities emerge. All curvature invariants assume constant values apart from the GB combination that is vanishing at exactly $r=r_{h}$.

We have performed a numerical integration of the system (30) to determine the solutions for the metric function $B$ and scalar field $\phi$ in the whole radial regime. To this end, we have used Eqs. (37) and (38) as boundary conditions, and finally integrated Eq. (29) with a randomly chosen boundary condition $e^{A\left(r_{h}\right)}=2$. In Figs. 1(a) and 1(b), we depict the profiles of the two metric components $-g_{t t}$ and $g_{r r}$, respectively, for a variety of forms of the coupling function $f(\phi)$. We observe that the qualitative behavior of the two metric components remains largely unaffected by the exact form of $f(\phi)$, especially at the small- $r$ regime. As our analytic calculations revealed, the $g_{r r}$ component diverges as $r \rightarrow r_{h}$ but the $g_{t t}$ remains finite thus failing to adopt a black-hole profile. We also note that the behavior of the two metric functions at asymptotic infinity fail to describe an asymptotically flat spacetime: the $g_{r r}$ metric component approaches a constant value different from unity whereas the $g_{t t}$ component diverges as $\rightarrow \infty$. This is due to the fact that the GB term cannot by itself support a robust solution at asymptotic infinity since it is subdominant to the Ricci curvature in that regime. Had we found a black-hole solution with a regular horizon at small values of $r$ supported only by the GB term, this would have been smoothly matched to a solution of the complete Einsteinscalar-GB theory for large values of $r$.

The regularity of the spacetime, despite the divergence of the $g_{t t}$ component at large distances, as well as the domain of dominance of the GB term are clearly reflected in Fig. 2: this scalar gravitational quantity vanishes very close to and far away from $r_{h}$ while having nontrivial values at small and intermediate distances.

The solution for the scalar field $\phi$ is presented in Fig. 3(a). As clearly shown, it adopts finite constant values ${ }^{1}$ asymptotic form near $r_{h}$ and at asymptotic infinity which leads to its first

\footnotetext{
${ }^{1}$ Instead of solving the set of field equations as an initial value problem, a shooting method could be applied in order to obtain solutions with a vanishing scalar field at infinity. However, this could be applied only for the scalar field and not for the metric functions for which the correct asymptotic form is obtained only after reinstating the Ricci scalar in the theory.
} 


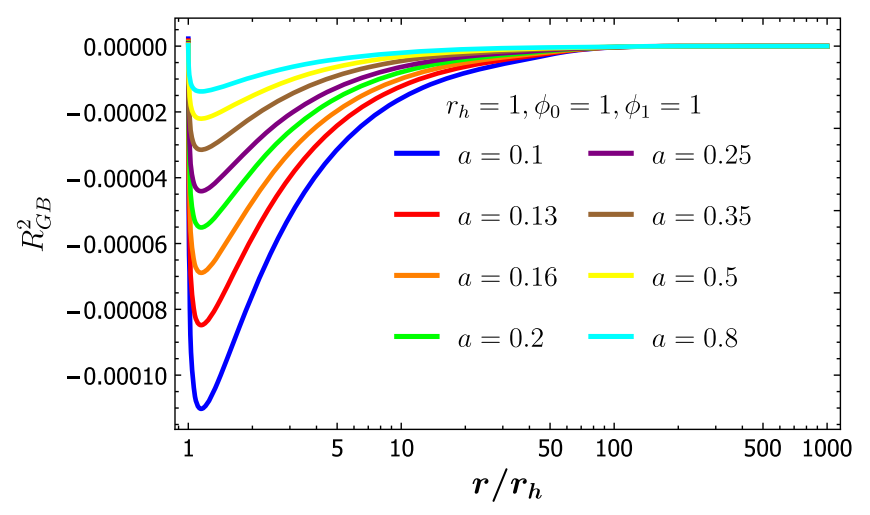

FIG. 2. The Gauss-Bonnet term $R_{\mathrm{GB}}^{2}$ for a pure scalar-GaussBonnet solution and a linear coupling function $f(\phi)=a \phi$, for various values of $a$.

derivative having a vanishing value at both of these regimes. This profile results into the form of the contribution of the scalar kinetic term to the effective energy-momentum tensor that is shown in Fig. 3(b): as expected, this contribution has a nonzero value at intermediate values of the radial coordinate where the scalar field has a nontrivial profile. The same is true for the contribution of the GB term to the total energymomentum tensor (5): this is again nontrivial at intermediate distances, in accordance to Fig. 2, and has exactly the same form as the $T_{\phi_{t}}^{t}$ contribution, depicted in Fig. 3(b), but with the exact opposite sign: it is this behavior that guarantees the vanishing total energy-momentum tensor as our analysis demanded. Let us stress that both families of solutions derived in this section correspond to nontrivial gravitational solutions with finite, zero total energy-momentum tensor whose physical significance will be studied elsewhere.

In the aforementioned numerical analysis, we have considered only positive values for the coupling constant $\alpha$, which appears in the expression of the coupling function $f(\phi)$. However, in the context of a generalized gravitational theory, negative values of $\alpha$ could be also considered.

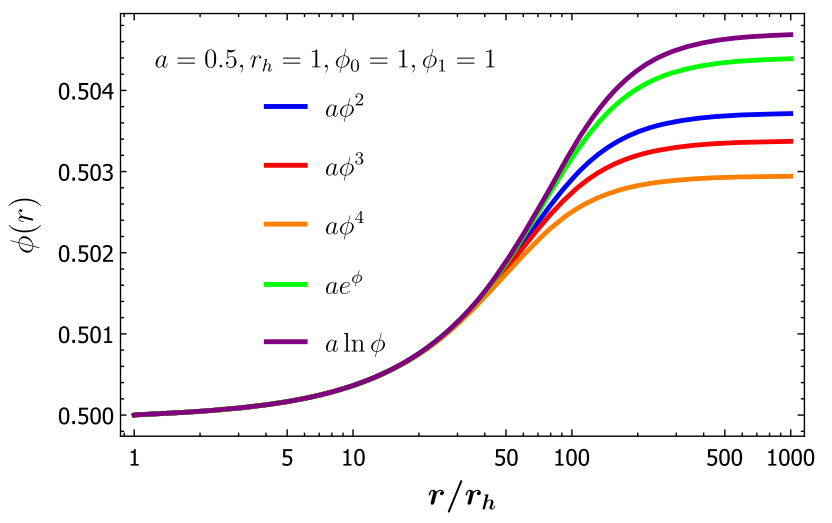

(a)
In fact, black-hole solutions with scalar hair do emerge in the complete Einstein-scalar-GB theory with negative $\alpha$ $[38,68]$. In the present, pure scalar-GB theory, we therefore considered negative values of $\alpha$, too. According to the results we obtained, solutions with a nontrivial scalar field emerge only if the sign of $\phi_{1}$, associated with the first derivative of the scalar field near $r_{h}$, is also reversed. However, the obtained solutions have the same characteristics as the ones derived for $\alpha>0$ and thus do not describe black-hole solutions.

In a final attempt to find black-hole solutions, we have also considered the case of an exotic scalar field with the opposite sign in front of its kinetic term. The addition of the GB term in the Einstein-scalar theory has the advantage of producing novel solutions, such as black holes with scalar hair or wormholes, without the need of any exotic matter. However, in the context of the pure scalar-GB theory, where black holes with scalar hair fail to emerge, an exotic scalar field could provide an interesting alternative. According to our calculations, the near- $r_{h}$ solutions for the scalar field and the $g_{r r}$ component in this case remain the same, and only the sign of the second term of the metric function $A(r)$ in Eq. (39), or equivalently the sign of the parameter $\phi_{1}$, is reversed. Once again, no black-hole solutions emerge since the $g_{t t}$ component takes on a constant value, as $r \rightarrow r_{h}$, instead of a vanishing one.

In Sec. VI, we will return to the study of all the aforementioned cases of the pure scalar-GB theory in order to investigate the deeper reason for failing to obtain blackhole solutions.

\section{ALTERNATIVE SPACETIME LINE ELEMENTS}

The main objective of this work is to find an asymptotic solution that describes a regular black-hole horizon. The line element (1) employed in the previous section has failed to accomplish this task. While retaining the

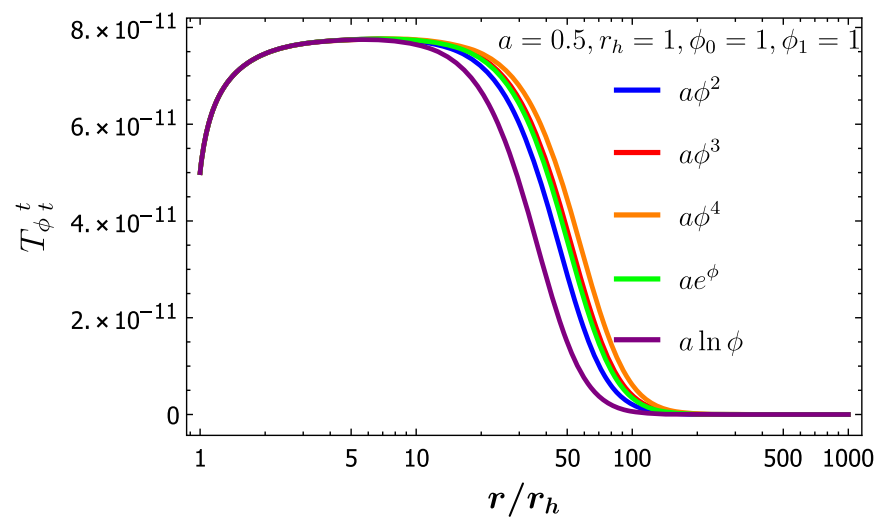

(b)

FIG. 3. (a) The scalar field $\phi$ and (b) the $(t t)$ component of the scalar kinetic-term contribution to the effective energy-momentum tensor, for various forms of the coupling function. 
assumptions of staticity and spherical symmetry, we could consider alternative ansatzes for the line element of the spacetime that could perhaps allow for more general families of solutions. To this end, we consider the following line element:

$$
d s^{2}=-e^{A(r)} d t^{2}+e^{B(r)} d r^{2}+H^{2}(r)\left(d \theta^{2}+\sin ^{2} \theta d \varphi^{2}\right) .
$$

Note that, by setting $H(r)=r$, we recover the line element employed in the previous section. A line element of this general form was used in the context of the Einstein-scalarGB theory in order to find traversable wormhole solutions [136] - there, it was demonstrated that the above line element led to more extended families of solutions even in cases where the line element (1) did not allow for any solutions at all. It seems therefore justified that such a line element could be used in our quest for black-hole solutions, too.

Employing then the line element (46), we find the following explicit expressions for the components of the gravitational field equations:

$$
\begin{aligned}
T^{t}{ }_{t}= & \frac{e^{-2 B}}{4 H^{2}}\left[8 \dot { f } \left(B^{\prime} \phi^{\prime}\left(e^{B}-3 H^{\prime 2}\right)\right.\right. \\
& \left.-2 e^{B} \phi^{\prime \prime}+2 H^{\prime 2} \phi^{\prime \prime}+4 H^{\prime} H^{\prime \prime} \phi^{\prime}\right) \\
& \left.+\phi^{\prime 2}\left(-16 \ddot{f}\left(e^{B}-H^{\prime 2}\right)-e^{B} H^{2}\right)\right]=0 \\
T^{r}{ }_{r}= & \frac{e^{-2 B} \phi^{\prime}}{4 H^{2}}\left[e^{B} H^{2} \phi^{\prime}-8 \dot{f} A^{\prime}\left(e^{B}-3 H^{\prime 2}\right)\right]=0 \\
T^{\theta}{ }_{\theta}= & T^{\varphi}{ }_{\varphi}=\frac{e^{-2 B}}{4 H}\left[+4 \dot{f}\left(2 A^{\prime \prime} H^{\prime} \phi^{\prime}\right.\right. \\
& \left.+A^{\prime}\left(-3 B^{\prime} H^{\prime} \phi^{\prime}+2 H^{\prime \prime} \phi^{\prime}+2 H^{\prime} \phi^{\prime \prime}\right)+\left(A^{\prime}\right)^{2} H^{\prime} \phi^{\prime}\right) \\
& \left.+\phi^{\prime 2}\left(8 A^{\prime} \ddot{f} H^{\prime}-e^{B} H\right)\right]=0,
\end{aligned}
$$

and the equation for the scalar field

$$
\begin{aligned}
& \frac{4 \dot{f} e^{-B}}{H}\left[-4 \dot{f}\left(2 A^{\prime \prime}\left(e^{B}-H^{\prime 2}\right)\right.\right. \\
& \left.\left.\quad-A^{\prime}\left(B^{\prime}\left(e^{B}-3 H^{2}\right)+4 H^{\prime} H^{\prime \prime}\right)+A^{\prime 2}\left(e^{B}-H^{\prime 2}\right)\right)\right] \\
& \quad+2 H \phi^{\prime \prime}+4 H^{\prime} \phi^{\prime}+H A^{\prime} \phi^{\prime}-H B^{\prime} \phi^{\prime}=0 .
\end{aligned}
$$

We may, as before, work first with the limit $e^{A} \rightarrow 0$, or $A^{\prime} \rightarrow \infty$, as $r \rightarrow r_{h}$. Then, Eq. (48) may be solved to yield an expression for the dependent function $e^{B}$ :

$$
e^{B}=\frac{24 \dot{f} A^{\prime} H^{\prime 2}}{8 \dot{f} A^{\prime}-H^{2} \phi^{\prime}}
$$

From the above, we may again find an expression for $B$ that, together with the one for $e^{B}$, could be used to eliminate the metric function $B$ from the remaining field equations.
These, then, reduce to a set of three, coupled, second-order, ordinary differential equations for the metric functions $A$ and $H$ and the scalar field $\phi$, which may be written as ${ }^{2}$

$$
A^{\prime \prime}=\frac{\tilde{P}}{4 \dot{f} \tilde{S}}, \quad \phi^{\prime \prime}=\frac{\phi^{\prime} \tilde{Q}}{\tilde{S}}, \quad H^{\prime \prime}=\frac{\tilde{K}}{4 \dot{f} \tilde{S}} .
$$

In order to reduce the technicalities of our analysis, we present the expression for $B^{\prime}$ and the explicit forms of the functions $\tilde{S}, \tilde{P}, \tilde{Q}$ and $\tilde{K}$ in Appendix B.

Let us instead focus on the physical implications of this system of equations. In the limit $A^{\prime} \rightarrow \infty$ while $H, \phi$ and $\phi^{\prime}$ remain finite, we obtain

$$
e^{B}=3 H^{2}+\mathcal{O}\left(\frac{1}{A^{\prime}}\right)
$$

By replacing the above in Eq. (52), we get near the point of interest, $r \simeq r_{h}$, the results

$$
\begin{aligned}
\phi^{\prime \prime} & =\frac{1}{8} \phi^{\prime} A^{\prime}+\mathcal{O}(1), \\
A^{\prime \prime} & =-\frac{3}{8} A^{\prime 2}+\mathcal{O}\left(A^{\prime}\right), \\
H^{\prime \prime} & =\frac{1}{8} H^{\prime} A^{\prime}+\mathcal{O}(1) .
\end{aligned}
$$

Again, in order to keep $\phi^{\prime \prime}$ finite we demand that $\phi^{\prime}\left(r_{h}\right)=0$. Integrating twice the last two equations with respect to the radial coordinate, we obtain the asymptotic solutions,

$e^{A}=a_{1}\left(r-r_{h}\right)^{8 / 3}+\cdots, \quad H=h_{1}\left(r-r_{h}\right)^{4 / 3}+\cdots$,

where $a_{1}$ and $h_{1}$ are integration constants. We observe that the metric function $e^{A}$ vanishes indeed at $r=r_{h}$, as expected near a black-hole horizon. However, the circumferential radius $H^{2}(r)$ also vanishes at the same point thus signaling the presence of an additional pathology in the coordinate system. If we define a new radial coordinate as $l \equiv h_{1}\left(r-r_{h}\right)^{4 / 3}$, then the line element (46) becomes

$$
d s^{2}=-e^{\tilde{A}} d t^{2}+e^{\tilde{B}} d l^{2}+l^{2}\left(d \theta^{2}+\sin ^{2} \theta d \varphi^{2}\right),
$$

with $e^{\tilde{A}} \sim a_{2} l^{2}+\cdots$ and $e^{\tilde{B}} \sim b_{0}=$ const. We may thus conclude that the obtained asymptotic solution is again not a black hole but more likely a particlelike solution with similar characteristics as the ones derived in Sec. III A.

Let us now use the alternative condition that, as $r \rightarrow r_{h}$, we have $e^{B} \rightarrow \infty$, or equivalently $B^{\prime} \rightarrow-\infty$.

\footnotetext{
${ }^{2}$ This holds under the assumption that $H^{\prime \prime} \neq 0$, which corresponds to the case $H(r)=r$ studied in the previous subsections.
} 
As in Sec. III B, we may then solve Eq. (48) with respect to $A^{\prime}$, obtaining the result

$$
A^{\prime}=\frac{H^{2} e^{B} \phi^{\prime}}{8\left(e^{B}-3 H^{\prime 2}\right) \dot{f}} .
$$

For a regular black-hole spacetime, we will demand that both the scalar field $\phi$ and the circumferential radius $H$ remain finite near the horizon. Then, there are two distinct cases:

(i) If $H^{\prime}$ remains finite near $r_{h}$, then, in the limit $e^{B} \rightarrow \infty$, Eq. (59) leads to

$$
A^{\prime} \simeq \frac{H^{2} \phi^{\prime}}{8 \dot{f}}+\mathcal{O}\left(e^{-B}\right)
$$

If, as assumed, $H^{2}, \phi$ and $\phi^{\prime}$ adopt constant, finite values at the black-hole horizon, then $A \simeq a_{0}+$ $a_{1}\left(r-r_{h}\right)+\cdots$ and the metric component $e^{A}$ adopts a constant, instead of a vanishing, value at $r \simeq r_{h}$.

(ii) If $H^{\prime}$ is allowed to diverge, then a more careful analysis should be performed. As an indicative example, we may consider the "conformal" case $H=r e^{B / 2}$, which was used in [136]. Then, Eq. (59) becomes

$$
A^{\prime}=-\frac{e^{B} r^{2} \phi^{\prime}}{2 \dot{f}\left(8+12 r B^{\prime}+3 r^{2} B^{\prime 2}\right)} .
$$

For the conventional black-hole dependence where $e^{B} \simeq b_{1}\left(r-r_{h}\right)+\cdots$, the aforementioned equation gives $A^{\prime} \rightarrow 0$, thus leading to a constant value for the $g_{t t}$ component near $r_{h}$. For an alternative dependence, such as the logarithmic given in Eq. (37), we obtain instead that $A^{\prime} \rightarrow$ const, which however leads again to a constant $g_{t t}$ at $r \simeq r_{h}$.

Therefore, even in the context of the more general line element (46), the condition $e^{B} \rightarrow \infty$ leads to a solution with similar characteristics as the ones that emerged in Sec. III B. Overall, the system of field equations fails to admit an asymptotic black-hole solution with the anticipated behavior (12) for both the $g_{t t}$ and $g_{r r}$ metric components.

\section{SOLUTIONS WITH HORIZONS WHEN $\Lambda \neq 0$}

In this section, we reinstate the cosmological constant $\Lambda$ in order to investigate its role in the emergence or not of solutions with a horizon in the context of the pure scalarGauss-Bonnet theory. For $\Lambda \neq 0$, Eq. (9) takes the form of a polynomial with respect to the metric function $e^{B}$, i.e., $\alpha e^{2 B}+\beta e^{B}+\gamma=0$. This may be solved to yield

$$
e^{B}=\frac{-\beta \pm \sqrt{\beta^{2}-4 \alpha \gamma}}{2 \alpha},
$$

where

$\alpha=-r^{2} \Lambda V, \quad \beta=\frac{r^{2} \phi^{\prime 2}}{4}-2 \dot{f} \phi^{\prime} A^{\prime}, \quad \gamma=6 \dot{f} \phi^{\prime} A^{\prime}$.

Taking the derivative of the above expression with respect to the radial coordinate, we may eliminate $B^{\prime}$ from the field equations replacing it by

$$
B^{\prime}=-\frac{\gamma^{\prime}+\beta^{\prime} e^{B}+\alpha^{\prime} e^{2 B}}{2 \alpha e^{2 B}+\beta e^{B}} .
$$

The field equations then reduce to a system of two coupled, second-order, ordinary differential equations for $A$ and $\phi$. This system now has the form

$$
A^{\prime \prime}=-\frac{P_{1}}{S_{1}}, \quad \phi^{\prime \prime}=-\frac{Q_{1}}{4 S_{1}} \phi^{\prime},
$$

where

$$
\begin{aligned}
P_{1}= & e^{B}\left(-17 r^{2} A^{\prime 2} \phi^{\prime 3} \dot{f}+64 A^{\prime 3} \phi^{\prime 2} \dot{f}^{2}+18 r A^{\prime} \phi^{\prime 3} \dot{f}\right)+e^{2 B}\left(-8 r^{2} A^{\prime} \phi^{\prime 4} \ddot{f}+9 r^{2} A^{\prime 2} \phi^{\prime 3} \dot{f}\right. \\
& \left.-20 \Lambda r^{2} A^{\prime 2} \phi^{\prime} \dot{f}-8 r A^{\prime} \phi^{\prime 3} \dot{f}-24 A^{\prime 3} \phi^{\prime 2} \dot{f}^{2}+72 \Lambda r A^{\prime} \phi^{\prime} \dot{f}-r^{4} A^{\prime} \phi^{\prime 4}+2 r^{3} \phi^{\prime 4}\right) \\
& \times e^{3 B}\left(4 \dot{f} \Lambda r^{2} A^{\prime 2} \phi^{\prime}+6 \dot{f} r A^{\prime} \phi^{\prime 3}-96 \dot{f} \Lambda r A^{\prime} \phi^{\prime}-2 \Lambda r^{4} A^{\prime} \phi^{\prime 2}-r^{3} \phi^{\prime 4}+4 \Lambda r^{3} \phi^{\prime 2}\right) \\
& +e^{4 B}\left(24 \dot{f} \Lambda r A^{\prime} \phi^{\prime}+8 \Lambda^{2} r^{4} A^{\prime}-16 \Lambda^{2} r^{3}\right)+16 e^{5 B} \Lambda^{2} r^{3}-72 \dot{f}^{2} A^{\prime 3} \phi^{\prime 2}, \\
Q= & e^{B}\left(36 \dot{f} r^{2} A^{\prime} \phi^{\prime 3}+896 \dot{f} A^{\prime} \ddot{f} \phi^{\prime 3}-192 \dot{f}^{2} A^{\prime 2} \phi^{\prime 2}-16 r^{2} \ddot{f} \phi^{\prime 4}-120 \dot{f} r \phi^{\prime 3}\right) \\
& +e^{2 B}\left(-20 \dot{f} r^{2} A^{\prime} \phi^{\prime 3}+144 \dot{f} \Lambda r^{2} A^{\prime} \phi^{\prime}-320 \dot{f} A^{\prime} \ddot{f} \phi^{\prime 3}+32 \dot{f}^{2} A^{\prime 2} \phi^{\prime 2}\right. \\
& \left.+16 r^{2} \ddot{f} \phi^{\prime 4}+128 \Lambda r^{2} \ddot{f} \phi^{\prime 2}+64 \dot{f} r \phi^{\prime 3}-96 \dot{f} \Lambda r \phi^{\prime}+r^{4} \phi^{\prime 4}\right) \\
& +e^{3 B}\left(-80 \dot{f} \Lambda r^{2} A^{\prime} \phi^{\prime}-128 \Lambda r^{2} \ddot{f} \phi^{\prime 2}-8 \dot{f} r \phi^{\prime 3}+128 \dot{f} \Lambda r \phi^{\prime}-4 \Lambda r^{4} \phi^{\prime 2}\right) \\
& +e^{4 B}\left(-32 \dot{f} \Lambda r \phi^{\prime}-32 \Lambda^{2} r^{4}\right)-576 \dot{f} A^{\prime} \ddot{f} \phi^{\prime 3}+288 \dot{f}^{2} A^{\prime 2} \phi^{\prime 2}, \\
S_{1}= & 8 \dot{f} \phi^{\prime}\left[2 \dot{f} A^{\prime} \phi^{\prime}\left(-14 e^{B}+5 e^{2 B}+9\right)-e^{B}\left(e^{B}-2\right) r^{2} \phi^{\prime 2}+4 e^{2 B}\left(e^{B}-1\right) \Lambda r^{2}\right] .
\end{aligned}
$$


We will assume that, near the black-hole horizon $r_{h}$, $e^{A} \rightarrow \infty$, or equivalently $A^{\prime} \rightarrow \infty$. Then, Eq. (62) takes the expanded form:

$e^{B}=\frac{-2 \dot{f} \phi^{\prime}}{r^{2} \Lambda V} A^{\prime}+\frac{-24 \Lambda V \dot{f} \phi^{\prime}+2 \dot{f} \phi^{\prime 3}}{8 \Lambda V \dot{f} \phi^{\prime}}+\mathcal{O}\left(\frac{1}{A^{\prime}}\right)$.

If we replace the above expansion, too, into the system (65), we obtain the following equations in the limit $A^{\prime} \rightarrow \infty$ :

$$
\begin{aligned}
& A^{\prime \prime}=-\frac{2 \dot{f} \phi^{\prime}}{r^{3} \Lambda V} A^{\prime 2}+\mathcal{O}\left(A^{\prime}\right), \\
& \phi^{\prime \prime}=\left(1-\frac{2 \dot{f} \phi^{\prime}}{r^{3} \Lambda V}\right) \phi^{\prime} A^{\prime}+\mathcal{O}(1) .
\end{aligned}
$$

The finiteness of $\phi^{\prime \prime}$ at the horizon dictates that the following relation should hold:

$$
\phi_{h}^{\prime}=\frac{r_{h}^{3} \Lambda}{2 \dot{f}\left(\phi_{h}\right)} .
$$

The above condition on the first derivative of the scalar field at the horizon is analogous to the one derived in the context of the Einstein-scalar-Gauss-Bonnet theory-there, that condition also related $\phi_{h}^{\prime}$ to the parameters of the theory and ensured the regularity of the black-hole horizon. Here, we observe that the condition (72) relates $\phi^{\prime}$ with the value of the cosmological constant.

Let us proceed to derive the complete form of the asymptotic solution near $r_{h}$. By using the above condition on $\phi_{h}^{\prime}$, the system (70) and (71) takes the simplified form:

$$
\begin{aligned}
& A^{\prime \prime}=-A^{\prime 2}+\mathcal{O}\left(A^{\prime}\right), \\
& \phi^{\prime \prime}=\mathcal{O}(1) .
\end{aligned}
$$

Again, this resembles the behavior obtained around a black-hole horizon. Integrating Eq. (73) with respect to $r$, we obtain the usual behavior

$$
A^{\prime}=\frac{1}{r-r_{h}},
$$

while a second integration leads to the complete asymptotic solution for the metric functions and the scalar field near $r_{h}$ :

$$
\begin{aligned}
e^{A} & =a_{1}\left(r-r_{h}\right)+\cdots, \\
e^{-B} & =b_{1}\left(r-r_{h}\right)+\cdots, \\
\phi & =\phi_{h}+\phi_{h}^{\prime}\left(r-r_{h}\right)+\phi_{h}^{\prime \prime}\left(r-r_{h}\right)^{2}+\cdots .
\end{aligned}
$$

The above describes indeed a solution with a horizon and a regular scalar field. There is, however, a caveat: if we replace $\phi_{h}^{\prime}$ from Eq. (72) into Eq. (69), we find

$$
b_{1}=-1 / r_{h} .
$$

The robustness of the metric is then ensured only if Eqs. (76)-(78) are rewritten as

$$
\begin{aligned}
e^{A} & =\left|a_{1}\right|\left(r_{c}-r\right)+\cdots, \\
e^{-B} & =\frac{1}{r_{c}}\left(r_{c}-r\right)+\cdots \\
\phi & =\phi_{c}+\phi_{c}^{\prime}\left(r_{c}-r\right)+\phi_{c}^{\prime \prime}\left(r_{c}-r\right)^{2}+\cdots .
\end{aligned}
$$

Note that $a_{1}$ is a completely arbitrary constant and thus may be appropriately chosen. The above reveal that, when $\Lambda>0$, the asymptotic solution we have found corresponds to a cosmological horizon $r_{c}$ with a nontrivial scalar field. Such solutions have indeed been derived in the context of the pure scalar-Gauss-Bonnet theory but from the perspective of a homogeneous, isotropic universe [146]. Here, we have in fact rederived those solutions describing a de Sitter universe using isotropic, spherically symmetric coordinates. In the case of an anti-de Sitter spacetime with $\Lambda<0$, no cosmological horizon exists and no robust black-hole solution emerges either in the context of our theory. Therefore, even in the presence of a nonvanishing cosmological constant, the pure scalar-Gauss-Bonnet theory fails to support a regular black-hole solution with scalar hair.

We close this section by noticing that the asymptotic solution (80)-(82) emerges also in the case where we work with the assumption that near the horizon it is $e^{B}$, or $B^{\prime}$, that diverges; this is expected for a physical, spherically symmetric horizon. Also, similar solutions which possess again a cosmological rather than a black-hole horizon emerge if we employ the more general line element (46) - as their characteristics are similar to the ones derived above, we refrain from presenting the corresponding analysis.

\section{SYNERGY BETWEEN THE RICCI AND GB TERMS}

In order to shed more light on the fact that the pure scalar-Gauss-Bonnet theory does not admit black-hole solutions but allows for cosmological solutions with a horizon to emerge, we turn to the complete Einstein-scalarGB theory. In the context of the latter, we will investigate the contribution of each gravitational term to the formation of a black hole. To this end, we choose the case of the exponential coupling function $f(\phi)=\alpha e^{-\phi}$, where $\alpha$ is a positive coupling constant. This choice leads to the dilatonic black holes that were found in [13] and studied again in [38]. They are a one-parameter family of 
black-hole solutions, with the independent parameter being any one of the three parameters of the theory, namely $\left(\alpha, r_{h}, \phi_{h}\right)$, due to the rescaling symmetries of the theory. Here, we fix the horizon radius $r_{h}$ to unity while the value of the scalar field at the horizon $\phi_{h}$ is determined by the boundary condition $\phi_{\infty}=1$ at infinity. These leave the coupling constant $\alpha$ as the independent parameter of the theory.

A theoretical argument for the regularity of the formed black-hole horizon, analogous to the one that led to Eq. (72), leads to the following condition on the first derivative of the scalar field at the horizon [38]:

$$
\phi_{h}^{\prime}=\frac{r_{h}}{4 \dot{f}_{h}}\left(-1 \pm \sqrt{1-\frac{96 \dot{f}_{h}^{2}}{r_{h}^{4}}}\right)
$$

The reality of the value of $\phi_{h}^{\prime}$ imposes the following additional constraint on the coupling function:

$$
\dot{f}_{h}^{2}<\frac{r_{h}^{4}}{96} .
$$

For the choice $f(\phi)=\alpha e^{-\phi}$, the above reduces to a constraint on the maximum allowed value of the coupling parameter, that is

$$
\frac{\alpha}{r_{h}^{2}} \simeq 0.123
$$

Black-hole solutions then arise in the context of the Einstein-scalar-GB theory for the range of values [0, 0.123] of the coupling constant $\alpha$. When $\alpha \rightarrow 0$, the GB term decouples from the theory and the only black-hole solution is the Schwarzschild solution characterized by a trivial scalar field. As $\alpha$ adopts a nonvanishing value, a GB black hole is formed that possesses scalar hair and a horizon radius smaller than the one of the Schwarzschild solution [38]. In Table I, we display a number of indicative values of $\alpha$ in the range [0, 0.123] and the values of the Ricci scalar and the combination $f(\phi) R_{\mathrm{GB}}^{2}$ near the horizon for each of the corresponding black-hole solutions. We observe that for small values of the coupling constant, the Ricci term adopts a very small value - this is due to the fact that, for small $\alpha$, the obtained solution is still very close to the Schwarzschild one that is a vacuum solution with $R=0$. The GB term, in contrast, adopts a much larger value from the beginning due to the contribution of the Riemann tensor, which is not zero even for the Schwarzschild solution. As $\alpha$ moves towards its maximum value, the curvature of spacetime increases and this enhances the magnitude of both gravitational terms. Above the maximum value $a_{\max }=0.123$, no black-hole solutions emerge.

We also observe that the sign of $R$ remains always positive, which reflects the positive curvature of spacetime around the formed horizon. In the Einstein-Hilbert action,
TABLE I. An indicative list of values of the coupling constant $\alpha$ and the values of the Ricci scalar $R$ and the combination $f(\phi) R_{\mathrm{GB}}^{2}$ near the black-hole horizon $r_{h}$.

\begin{tabular}{ccc}
\hline \hline$\alpha / r_{h}^{2}$ & $R$ & $f(\phi) R_{\mathrm{GB}}^{2}$ \\
\hline 0.001 & $3.318 \times 10^{-9}$ & 0.004 \\
0.006 & $5.002 \times 10^{-8}$ & 0.025 \\
0.015 & $1.536 \times 10^{-6}$ & 0.070 \\
0.025 & $1.112 \times 10^{-5}$ & 0.117 \\
0.037 & $6.134 \times 10^{-5}$ & 0.182 \\
0.051 & $2.835 \times 10^{-4}$ & 0.270 \\
0.065 & $9.790 \times 10^{-4}$ & 0.372 \\
0.075 & $2.051 \times 10^{-3}$ & 0.452 \\
0.087 & $4.897 \times 10^{-3}$ & 0.571 \\
0.097 & $9.722 \times 10^{-3}$ & 0.691 \\
0.106 & $1.962 \times 10^{-2}$ & 0.847 \\
0.116 & $4.120 \times 10^{-2}$ & 1.067 \\
0.123 & $8.097 \times 10^{-2}$ & 1.320 \\
\hline \hline
\end{tabular}

the presence of the Ricci term leads to the attractive force of gravity. The presence of the GB term, on the other hand, produces the opposite effect as it leads to a repulsive force in the theory. Indeed, if we examine the components of the effective energy-momentum tensor near the horizon, we obtain the following expressions for the effective energydensity and pressure components of the system:

$$
\begin{gathered}
\rho=-T^{t}{ }_{t}=-\frac{2 e^{-B}}{r^{2}} B^{\prime} \phi^{\prime} \dot{f}+\mathcal{O}\left(r-r_{h}\right), \\
p_{r}=T_{r}^{r}=-\frac{2 e^{-B}}{r^{2}} A^{\prime} \phi^{\prime} \dot{f}+\mathcal{O}\left(r-r_{h}\right), \\
p_{\theta}=T_{\theta}^{\theta}=\frac{2 e^{-2 B}}{r} A^{\prime 2} \phi^{\prime} \dot{f}+\mathcal{O}\left(r-r_{h}\right),
\end{gathered}
$$

whereas $p_{\varphi}=p_{\theta}$ due to the spherical symmetry. Note that the contribution of the scalar-field kinetic term vanishes near the horizon and the dominant contribution to the energy-momentum tensor components comes from its effective potential, i.e., by its coupling to the GB term. The combination $\phi^{\prime} \dot{f}$ is always negative at the horizon due to the regularity constraint (83). Also, near the horizon radius, it holds that $A^{\prime} \simeq-B^{\prime} \simeq 1 /\left(r-r_{h}\right)+\cdots$, since $e^{A}$ increases near the horizon, as $r$ increases, while $e^{B}$ decreases. Employing the above, we are led to the final results:

$$
\begin{aligned}
& p_{r}=-\rho=\frac{2 b_{1}}{r_{h}^{2}}\left|\left(\phi^{\prime} \dot{f}\right)_{h}\right|>0, \\
& p_{\theta}=p_{\varphi}=-\frac{2 b_{1}^{2}}{r_{h}}\left|\left(\phi^{\prime} \dot{f}\right)_{h}\right|<0 .
\end{aligned}
$$

Therefore, the coupling between the scalar field and the GB term produces a negative effective energy-density and an 
equal in magnitude, but positive, radial pressure component at the horizon. In addition, the tangential pressure components $p_{\theta}=p_{\varphi}$ come out to be negative at the black-hole horizon. Clearly, the scalar-Gauss-Bonnet coupling violates the weak, dominant and strong energy conditions. It is this violation of the energy conditions that causes the evasion of the novel no-hair theorem [9] and allows for the emergence of black holes with nontrivial scalar hair $[13,38]$. The positive, outward radial pressure signifies in addition the repulsive role of the GB term in the system.

We may, therefore, interpret now the nonemergence of a black-hole horizon in the context of the pure scalar-GB theory. The presence of the Ricci term is necessary in order to provide the attractive force that will create the positively curved topology around the formed black hole. Even in vacuum, the Ricci term causes the formation of a black hole in the form of the Schwarzschild solution. When the GB term is turned on, the Schwarzschild solution gets naturally scalarized $^{3}$ as it is automatically donned with a nontrivial, regular scalar field. Due to the repulsive effect of the GB term, the black-hole horizon is now formed at a smaller horizon value than in the Schwarzschild case-indeed, in [38], it was demonstrated that, for a fixed coupling constant, a GB black hole has always a smaller horizon radius compared to the one of the Schwarzschild solution with the same mass. Apparently, gravity dominates over a smaller regime of spacetime, creating a black-hole topology, in the presence of the GB term. In other words, the same amount of mass needs to be "squeezed" more to create a black hole when the repulsive GB term is present in the theory.

Therefore, the GB term makes the formation of a black hole more difficult. What it does facilitate is the dressing of the black-hole solution with a nontrivial scalar field, a feature that would have been forbidden in its absence. As the value of the coupling constant increases, the weight of the GB term in the theory gradually increases, too. The same holds for its repulsive effect. Beyond the maximum value (85) of the GB coupling parameter, no black-hole horizon can be formed - or sustained - any more. We may easily then justify the fact that a pure scalar-GB theory cannot, in the absence of the Ricci scalar, create by itself a black-hole solution.

At the end of Sec. II, we considered also the case of a negative coupling constant $\alpha$, and failed again to produce viable black-hole solutions. As we saw, upon reversing the sign of $\alpha$ in the theory, solutions with a nontrivial scalar field emerge only if the sign of $\phi_{1}$ is also reversed. Then, through Eq. (45), the overall sign of the GB term remains the same, i.e., negative, leading again to a repulsive force

\footnotetext{
${ }^{3}$ This process differs from the one of spontaneous scalarization, which takes place when a tachyonic scalar mode causes the system to shift from the unstable Schwarzschild solution to a more stable solution with a nontrivial scalar field. This scalarization is realized only for a particular regime of the independent parameter where the Schwarzschild solution is destabilized.
}

that cannot support by itself a black-hole horizon. We also considered the case of an exotic scalar field with the opposite sign in front of its kinetic term. We then found similar solutions to the ones following for a physically acceptable scalar field but with the sign of the parameter $\phi_{1}$ reversed. In this case, and with all the other parameters remaining unchanged, the sign of the GB term is also reversed leading to an attractive gravitational force similar to the one described by the Ricci scalar. Yet, no black-hole solution was found. The deeper reason for this is the fact that, now, it is the exotic scalar field that exerts a repulsive force, which once again destroys the black-hole horizon.

In the case of cosmological solutions, the presence of the GB term in the theory leads to the emergence of singularityfree $[143,145,146]$ and inflationary solutions [146]. In a cosmological context, traditional gravity, in the form of the Ricci term, leads to the formation of the initial singularity, a feature that is not desirable in the theory. The addition of the GB term with its repulsive effect manages to provide the necessary outward pressure to the system so that the initial singularity is avoided, and a smooth transition between a collapsing and an expanding phase of the Universe is realized $[143,145,146]$. In the emergence of de Sitter, inflationary solutions [146], the GB term is again providing the outward pressure component that accelerates the expansion of the Universe even in the absence of any potential for the scalar field.

\section{CONCLUSIONS}

After the derivation, in an analytical way, of cosmological singularity-free and inflationary solutions in the context of the pure scalar-Gauss-Bonnet theory [146], here, we have investigated whether black-hole solutions can be supported in the context of the same theory. We have therefore ignored the presence of all terms associated with the Ricci term in the field equations and used both analytical and numerical means to integrate them. Any solutions that could emerge would rely solely on the synergy between the scalar field and the GB term in the theory and would correspond to zero-energy and zeropressure gravitational solutions.

We initially focused on the derivation of static, spherically symmetric solutions that would describe a regular black hole with a nontrivial scalar hair. In Sec. III, we solved analytically the field equations near the sought-for black-hole horizon as it is only there that ignoring the Ricci scalar, compared to the quadratic GB term, may be justified. Working with the assumption that there the $g_{t t}$ metric component vanishes, we derived a family of gravitational solutions with a finite $g_{r r}$ and a regular scalar field. The spacetime possesses a true singularity but a finite energy-momentum tensor, a profile that resembles the one of particlelike solutions in quadratic gravitational theories [137,139]. Alternatively, demanding that the $g_{r r}$ diverges at a specific value of the radial coordinate, we determined, 
first analytically and then numerically, a second family of gravitational solutions with no spacetime singularity and a finite, again, energy-momentum tensor. We have postponed the study of these two families of solutions and their physical interpretation for a future study.

Coming back to our quest for black-hole solutions, in Sec. IV, we considered a more general line element for the spacetime, which preserved the assumptions of staticity and spherical symmetry. Despite the increased flexibility of the line element, which resulted in the addition of a third unknown metric function, no solutions with a black-hole horizon were found. The asymptotic solutions derived analytically in the small- $r$ regime shared the same characteristics as the ones found in Sec. III.

Reinstating the cosmological constant, which was ignored in the first part of our analysis, we looked again for solutions with a horizon. The analysis in this case resembled the one that led to the derivation of regular black-hole solutions with scalar hair [38]. Indeed, solutions with a horizon and a regular scalar field were successfully found in Sec. V in the case of a positive cosmological constant, however, these were shown to correspond to a cosmological rather than to a black-hole horizon.

In Sec. VI, we looked more carefully at the synergy between the Ricci and GB terms. The values of the components of the energy-momentum tensor near the black-hole horizon reveal the repulsive effect of the GB term as opposed to the attractive effect of the Ricci term. As the latter is necessary in order to create the positive curvature around a black hole, no such solution emerges in the presence of only the GB term, which in fact works towards pushing outwards any distribution of matter. The same results follow also in the case where the coupling constant $\alpha$ is assumed to take negative, instead of positive, values since the GB term retains its overall negative sign, and thus its repulsive effect. Even in the context of the complete Einstein-scalar-GB theory, black holes emerge only up to a maximum value of the GB coupling parameter-it is only over this restricted parameter regime that the Ricci term manages to form a black-hole horizon despite the presence of the GB term. Nevertheless, the GB term justifies its presence by supporting a nontrivial, regular scalar field, a feature forbidden by general relativity. When an exotic scalar field is introduced in the theory instead of a physical one, the GB term reverses its sign exerting an attractive force, however, it is the exotic scalar field that now destroys the black-hole horizon with its own negative energy.

In the context of the effective-field-theory point of view, one could imagine adding even higher-derivative terms in the quadratic action (1). How are then the previously derived solutions $[38,131]$ modified by the presence of these gravitational corrections? In light of the analysis of Sec. VI, we conclude that this depends on the role of these terms when it comes to their contributions to the effective energy-momentum tensor. If such a higher-derivative term had a positive contribution to the radial pressure as the GB term, then it would have a destabilizing effect and blackhole solutions could emerge for a more restricted range of values for the coupling constant $\alpha$. If, on the other hand, the produced contribution to the radial pressure of the system was negative, the added term would have a role similar to that of the Ricci scalar and the emergence of black-hole solutions would be facilitated.

Closing this work, let us return to the question we posed in Sec. II to which we may now give a simple answer: we may indeed find spacetime regimes where the GB term is overwhelmingly dominant over the Ricci scalar, however, the gravitational solution that would form will not be a black hole. Nevertheless, solutions the existence of which does not rely on the attractive nature of the Ricci term, and thus on its presence in the theory, do exist and these include a plethora of interesting solutions such as particlelike solutions, cosmological solutions or even wormholes. As that part of the phase space of solutions of the pure scalarGB theory has not been adequately explored so far, we plan to perform such a comprehensive study soon.

\section{ACKNOWLEDGMENTS}

We would like to thank Naresh Dadhich and John Rizos for valuable discussions during the early stages of this work. This research is implemented through the Operational Program "Human Resources Development, Education and Lifelong Learning" and is cofinanced by the European Union (European Social Fund) and Greek national funds (MIS code: 5006022).

\section{APPENDIX A: SCALAR QUANTITIES}

By employing the metric components of the line element (1), one may compute the following scalar-invariant gravitational quantities:

$$
\begin{aligned}
R=+\frac{e^{-B}}{2 r^{2}}(4 & \left.e^{B}-4-r^{2} A^{\prime 2}+4 r B^{\prime}-4 r A^{\prime}+r^{2} A^{\prime} B^{\prime}-2 r^{2} A^{\prime \prime}\right), \\
R_{\mu \nu} R^{\mu \nu}= & +\frac{e^{-2 B}}{16 r^{4}}\left[8\left(2-2 e^{B}+r A^{\prime}-r B^{\prime}\right)^{2}\right. \\
& +r^{2}\left(r A^{\prime 2}-4 B^{\prime}-r A^{\prime} B^{\prime}+2 r A^{\prime \prime}\right)^{2} \\
& \left.+r^{2}\left(r A^{\prime 2}+A^{\prime}\left(4-r B^{\prime}\right)+2 r A^{\prime \prime}\right)^{2}\right], \\
R_{\mu \nu \rho \sigma} R^{\mu \nu \rho \sigma}= & +\frac{e^{-2 B}}{4 r^{4}}\left[r^{4} A^{\prime 4}-2 r^{4} A^{\prime 3} B^{\prime}-4 r^{4} A^{\prime} B^{\prime} A^{\prime \prime}\right. \\
& +r^{2} A^{\prime 2}\left(8+r^{2} B^{\prime 2}+4 r^{2} A^{\prime \prime}\right) \\
& \left.+16\left(e^{B}-1\right)^{2}+8 r^{2} B^{\prime 2}+4 r^{4} A^{\prime \prime 2}\right], \\
R_{\mathrm{GB}}^{2}=+\frac{2 e^{-2 B}}{r^{2}} & {\left[\left(e^{B}-3\right) A^{\prime} B^{\prime}-\left(e^{B}-1\right) A^{\prime 2}-2\left(e^{B}-1\right) A^{\prime \prime}\right] . }
\end{aligned}
$$




\section{APPENDIX B: GENERAL SYSTEM OF COUPLED EQUATIONS}

Employing the expression (51) for the metric function $e^{B}$, we may easily compute the first derivative of the metric function $B(r)$. This is found to have the form

$$
\begin{aligned}
B^{\prime}= & \frac{e^{-B}}{r \phi^{\prime}\left(H^{2} \phi^{\prime}-8 \dot{f} A^{\prime}\right)}\left\{-2 \phi^{\prime}\left[\phi^{\prime}\left(e^{B} H\left(r H^{\prime}+H\right)-4 r A^{\prime} \ddot{f}\left(e^{B}-3 H^{\prime 2}\right)\right)+e^{B} H^{2} r \phi^{\prime \prime}\right]\right. \\
& \left.+8 \dot{f}\left[r A^{\prime \prime} \phi^{\prime}\left(e^{B}-3 H^{\prime 2}\right)+r A^{\prime} \phi^{\prime \prime}\left(e^{B}-3 H^{\prime 2}\right)+2 A^{\prime} \phi^{\prime}\left(e^{B}-3 H^{\prime}\left(r H^{\prime \prime}+H^{\prime}\right)\right)\right]\right\} .
\end{aligned}
$$

Then, the field equations (47)-(50) reduce to a set of three, coupled, second-order, ordinary differential equations for the metric functions $A$ and $H$ and the scalar field $\phi$, given in Eq. (52). The functions $\tilde{S}, \tilde{P}, \tilde{Q}$ and $\tilde{K}$ have now the following explicit forms:

$$
\begin{aligned}
\tilde{S}= & 8 r\left[2 \dot{f} A^{\prime}\left(e^{B}-H^{\prime 2}\right)\left(e^{B}+3 H^{\prime 2}\right)+e^{B} H^{2} H^{\prime 2} \phi^{\prime}\right], \\
\tilde{P}= & 8 \dot{f}^{2} A^{\prime 2}\left(3 r A^{\prime}+4\right)\left(e^{B}-H^{\prime 2}\right)\left(e^{B}+3 H^{\prime 2}\right)+e^{B} H^{2} r \phi^{\prime 2}\left[-4 e^{B} H H^{\prime}\right. \\
& \left.\times A^{\prime}\left(16 \ddot{f}\left(e^{B}+H^{\prime 2}\right)+e^{B} H^{2}\right)\right]-4 e^{B} \dot{f} H A^{\prime} \phi^{\prime}\left[2 e^{B} r\left(H A^{\prime}+6 H^{\prime}\right)+H^{\prime 2}\left(H r A^{\prime}+4 r H^{\prime}-4 H\right)\right], \\
\tilde{Q}= & \phi^{\prime}\left[-4 e^{B} H^{\prime 2}\left(r A^{\prime}\left(8 \ddot{f}+H^{2}\right)+H\left(5 r H^{\prime}+H\right)\right)-e^{2 B} r\left(A^{\prime}\left(16 \ddot{f}+H^{2}\right)-4 H H^{\prime}\right)\right. \\
& \left.+48 r A^{\prime} \ddot{f} H^{\prime 4}\right]+2 \dot{f} A^{\prime}\left(r A^{\prime}-4\right)\left(e^{B}-H^{\prime 2}\right)\left(e^{B}+3 H^{\prime 2}\right), \\
\tilde{K}= & 8 \dot{f}^{2} A^{\prime} H^{\prime}\left(r A^{\prime}-4\right)\left(e^{B}-H^{\prime 2}\right)\left(e^{B}+3 H^{\prime 2}\right)+e^{B} H^{2} r H^{\prime} \phi^{\prime 2}\left[16 \ddot{f}\left(e^{B}-H^{\prime 2}\right)+e^{B} H^{2}\right] \\
& +4 e^{B} \dot{f} H \phi^{\prime}\left[-e^{B} r H^{\prime}\left(H A^{\prime}+12 H^{\prime}\right)+2 H^{\prime 3}\left(H r A^{\prime}+5 r H^{\prime}-2 H\right)+2 e^{2 B} r\right] .
\end{aligned}
$$

[1] https://www.ligo.org/.

[2] http://www.virgo-gw.eu/.

[3] K. S. Stelle, Phys. Rev. D 16, 953 (1977).

[4] T. P. Sotiriou, Lect. Notes Phys. 892, 3 (2015); E. Berti et al., Classical Quantum Gravity 32, 243001 (2015).

[5] J. D. Bekenstein, Phys. Rev. Lett. 28, 452 (1972); C. Teitelboim, Lett. Nuovo Cimento 3, 397 (1972).

[6] M. S. Volkov and D. V. Galtsov, JETP Lett. 50, 346 (1989); P. Bizon, Phys. Rev. Lett. 64, 2844 (1990); B. R. Greene, S. D. Mathur, and C. M. O’Neill, Phys. Rev. D 47, 2242 (1993); K. i. Maeda, T. Tachizawa, T. Torii, and T. Maki, Phys. Rev. Lett. 72, 450 (1994).

[7] H. Luckock and I. Moss, Phys. Lett. 176B, 341 (1986); S. Droz, M. Heusler, and N. Straumann, Phys. Lett. B 268, 371 (1991).

[8] J. D. Bekenstein, Ann. Phys. (N.Y.) 82, 535 (1974); 91, 75 (1975).

[9] J. D. Bekenstein, Phys. Rev. D 51, R6608 (1995).

[10] B. Zwiebach, Phys. Lett. 156B, 315 (1985).

[11] D. J. Gross and J. H. Sloan, Nucl. Phys. B291, 41 (1987).

[12] R. R. Metsaev and A. A. Tseytlin, Nucl. Phys. B293, 385 (1987).

[13] P. Kanti, N. E. Mavromatos, J. Rizos, K. Tamvakis, and E. Winstanley, Phys. Rev. D 54, 5049 (1996); 57, 6255 (1998).

[14] G. W. Gibbons and K. i. Maeda, Nucl. Phys. B298, 741 (1988).
[15] C. G. Callan, Jr., R. C. Myers, and M. J. Perry, Nucl. Phys. B311, 673 (1989).

[16] B. A. Campbell, M. J. Duncan, N. Kaloper, and K. A. Olive, Phys. Lett. B 251, 34 (1990); B. A. Campbell, N. Kaloper, and K. A. Olive, Phys. Lett. B 263, 364 (1991).

[17] S. Mignemi and N. R. Stewart, Phys. Rev. D 47, 5259 (1993).

[18] P. Kanti and K. Tamvakis, Phys. Rev. D 52, 3506 (1995).

[19] T. Torii, H. Yajima, and K. i. Maeda, Phys. Rev. D 55, 739 (1997).

[20] P. Kanti and K. Tamvakis, Phys. Lett. B 392, 30 (1997); P. Kanti and E. Winstanley, Phys. Rev. D 61, 084032 (2000).

[21] Z. K. Guo, N. Ohta, and T. Torii, Prog. Theor. Phys. 120, 581 (2008); K. i. Maeda, N. Ohta, and Y. Sasagawa, Phys. Rev. D 80, 104032 (2009); N. Ohta and T. Torii, Prog. Theor. Phys. 124, 207 (2010).

[22] B. Kleihaus, J. Kunz, and E. Radu, Phys. Rev. Lett. 106, 151104 (2011); B. Kleihaus, J. Kunz, S. Mojica, and E. Radu, Phys. Rev. D 93, 044047 (2016).

[23] P. Pani, C. F. B. Macedo, L. C. B. Crispino, and V. Cardoso, Phys. Rev. D 84, 087501 (2011); P. Pani, E. Berti, V. Cardoso, and J. Read, Phys. Rev. D 84, 104035 (2011).

[24] C. A. R. Herdeiro and E. Radu, Phys. Rev. Lett. 112, 221101 (2014).

[25] D. Ayzenberg and N. Yunes, Phys. Rev. D 90, 044066 (2014); 91, 069905(E) (2015).

[26] E. Winstanley, Lect. Notes Phys. 769, 49 (2009). 
[27] C. Charmousis, Lect. Notes Phys. 769, 299 (2009).

[28] C. A. R. Herdeiro and E. Radu, Int. J. Mod. Phys. D 24, 1542014 (2015).

[29] J. L. Blazquez-Salcedo et al., IAU Symp. 324, 265 (2017).

[30] G. W. Horndeski, Int. J. Theor. Phys. 10, 363 (1974).

[31] A. Nicolis, R. Rattazzi, and E. Trincherini, Phys. Rev. D 79, 064036 (2009).

[32] T. P. Sotiriou and V. Faraoni, Phys. Rev. Lett. 108, 081103 (2012).

[33] L. Hui and A. Nicolis, Phys. Rev. Lett. 110, 241104 (2013).

[34] T. P. Sotiriou and S. Y. Zhou, Phys. Rev. Lett. 112, 251102 (2014).

[35] E. Babichev and C. Charmousis, J. High Energy Phys. 08 (2014) 106.

[36] T. P. Sotiriou and S. Y. Zhou, Phys. Rev. D 90, 124063 (2014); R. Benkel, T. P. Sotiriou, and H. Witek, Classical Quantum Gravity 34, 064001 (2017); Phys. Rev. D 94, 121503 (2016).

[37] N. Yunes and L. C. Stein, Phys. Rev. D 83, 104002 (2011).

[38] G. Antoniou, A. Bakopoulos, and P. Kanti, Phys. Rev. Lett. 120, 131102 (2018); Phys. Rev. D 97, 084037 (2018).

[39] D. D. Doneva and S. S. Yazadjiev, Phys. Rev. Lett. 120, 131103 (2018).

[40] H. O. Silva, J. Sakstein, L. Gualtieri, T. P. Sotiriou, and E. Berti, Phys. Rev. Lett. 120, 131104 (2018).

[41] Y. Bardoux, M. M. Caldarelli, and C. Charmousis, J. High Energy Phys. 05 (2012) 054.

[42] K. Yagi, L. C. Stein, N. Yunes, and T. Tanaka, Phys. Rev. D 85, 064022 (2012); 93, 029902(E) (2016); D. Ayzenberg, K. Yagi, and N. Yunes, Phys. Rev. D 89, 044023 (2014).

[43] C. Charmousis, T. Kolyvaris, E. Papantonopoulos, and M. Tsoukalas, J. High Energy Phys. 07 (2014) 085.

[44] F. Correa, M. Hassaine, and J. Oliva, Phys. Rev. D 89, 124005 (2014).

[45] S. R. Dolan, S. Ponglertsakul, and E. Winstanley, Phys. Rev. D 92, 124047 (2015).

[46] J. L. Blazquez-Salcedo, C. F. B. Macedo, V. Cardoso, V. Ferrari, L. Gualtieri, F. S. Khoo, J. Kunz, and P. Pani, Phys. Rev. D 94, 104024 (2016).

[47] S. Bhattacharya and S. Chakraborty, Phys. Rev. D 95, 044037 (2017); I. Banerjee, S. Chakraborty, and S. SenGupta, Phys. Rev. D 96, 084035 (2017).

[48] D. D. Doneva and S. S. Yazadjiev, J. Cosmol. Astropart. Phys. 04 (2018) 011.

[49] H. Motohashi and M. Minamitsuji, Phys. Lett. B 781, 728 (2018); Phys. Rev. D 98, 084027 (2018).

[50] C. A. R. Herdeiro, E. Radu, N. Sanchis-Gual, and J. A. Font, Phys. Rev. Lett. 121, 101102 (2018); T. Delsate, C. Herdeiro, and E. Radu, Phys. Lett. B 787, 8 (2018); Y. Brihaye, C. Herdeiro, and E. Radu, Phys. Lett. B 788, 295 (2019).

[51] D. D. Doneva, S. Kiorpelidi, P. G. Nedkova, E. Papantonopoulos, and S. S. Yazadjiev, Phys. Rev. D 98, 104056 (2018).

[52] M. Butler, A. M. Ghezelbash, E. Massaeli, and M. Motaharfar, Mod. Phys. Lett. A 34, 1950232 (2019).

[53] B. Danila, T. Harko, F. S. N. Lobo, and M. K. Mak, Phys. Rev. D 99, 064028 (2019).

[54] M. M. Stetsko, arXiv:1811.05030.
[55] O. J. Tattersall, P. G. Ferreira, and M. Lagos, Phys. Rev. D 97, 084005 (2018).

[56] S. Mukherjee and S. Chakraborty, Phys. Rev. D 97, 124007 (2018).

[57] S. Chakrabarti, Eur. Phys. J. C 78, 296 (2018).

[58] E. Berti, K. Yagi, and N. Yunes, Gen. Relativ. Gravit. 50, 46 (2018).

[59] Y. Brihaye and B. Hartmann, Classical Quantum Gravity 35, 175008 (2018).

[60] K. Prabhu and L. C. Stein, Phys. Rev. D 98, 021503 (2018).

[61] Y. S. Myung and D.C. Zou, Phys. Rev. D 98, 024030 (2018); arXiv:1812.03604.

[62] J. L. Blazquez-Salcedo, D. D. Doneva, J. Kunz, and S. S. Yazadjiev, Phys. Rev. D 98, 084011 (2018); J. L. Blazquez-Salcedo, Z. A. Motahar, D. D. Doneva, F. S. Khoo, J. Kunz, S. Mojica, K. V. Staykov, and S. S. Yazadjiev, Eur. Phys. J. Plus 134, 46 (2019).

[63] R. Benkel, N. Franchini, M. Saravani, and T. P. Sotiriou, Phys. Rev. D 98, 064006 (2018).

[64] L. Iorio and M. L. Ruggiero, J. Cosmol. Astropart. Phys. 10 (2018) 021.

[65] J. Ovalle, R. Casadio, R. da Rocha, A. Sotomayor, and Z. Stuchlik, Europhys. Lett. 124, 20004 (2018); J. Ovalle, Phys. Lett. B 788, 213 (2019).

[66] L. Barack et al., Classical Quantum Gravity 36, 143001 (2019).

[67] Y.X. Gao, Y. Huang, and D. J. Liu, Phys. Rev. D 99, 044020 (2019).

[68] B. H. Lee, W. Lee, and D. Ro, Phys. Rev. D 99, 024002 (2019).

[69] H. Witek, L. Gualtieri, P. Pani, and T. P. Sotiriou, Phys. Rev. D 99, 064035 (2019).

[70] H. Motohashi and S. Mukohyama, Phys. Rev. D 99, 044030 (2019).

[71] J. Sultana and D. Kazanas, Gen. Relativ. Gravit. 50, 137 (2018).

[72] S. Nojiri, S. D. Odintsov, and V. K. Oikonomou, Phys. Rev. D 99, 044050 (2019).

[73] S. Qolibikloo and A. Ghodsi, Eur. Phys. J. C 79, 406 (2019).

[74] P. V. P. Cunha, C. A. R. Herdeiro, and E. Radu, Phys. Rev. Lett. 123, 011101 (2019).

[75] M. Minamitsuji and T. Ikeda, Phys. Rev. D 99, 044017 (2019); 99, 104069 (2019).

[76] M. M. Stetsko, Phys. Rev. D 99, 044028 (2019).

[77] Y. S. Myung and D.-C. Zou, Phys. Lett. B 790, 400 (2019).

[78] Y. Brihaye and L. Ducobu, Phys. Lett. B 795, 135 (2019).

[79] C. A. R. Herdeiro and E. Radu, Phys. Rev. D 99, 084039 (2019).

[80] T. Kobayashi, Rep. Prog. Phys. 82, 086901 (2019).

[81] H. O. Silva, C. F. B. Macedo, T. P. Sotiriou, L. Gualtieri, J. Sakstein, and E. Berti, Phys. Rev. D 99, 104041 (2019).

[82] A. de la Cruz-Dombriz and F. J. M. Torralba, J. Cosmol. Astropart. Phys. 03 (2019) 002.

[83] C.-Y. Wang, Y.-F. Shen, and Y. Xie, J. Cosmol. Astropart. Phys. 04 (2019) 022.

[84] P.-A. Cano and A. Ruiperez, J. High Energy Phys. 05 (2019) 189.

[85] F. M. Ramazanoglu, Phys. Rev. D 99, 084015 (2019). 
[86] P. G. S. Fernandes, C. A. R. Herdeiro, A. M. Pombo, E. Radu, and N. Sanchis-Gual, Classical Quantum Gravity 36, 134002 (2019).

[87] Y. Brihaye and B. Hartmann, Phys. Lett. B 792, 244 (2019).

[88] M. Saravani and T. P. Sotiriou, Phys. Rev. D 99, 124004 (2019).

[89] C. F. B. Macedo, J. Sakstein, E. Berti, L. Gualtieri, H. O. Silva, and T. P. Sotiriou, Phys. Rev. D 99, 104041 (2019).

[90] D. D. Doneva, K. V. Staykov, and S. S. Yazadjiev, Phys. Rev. D 99, 104045 (2019).

[91] A. Saffer, H. O. Silva, and N. Yunes, Phys. Rev. D 100, 044030 (2019).

[92] T. Anson, E. Babichev, C. Charmousis, and S. Ramazanov, J. Cosmol. Astropart. Phys. 06 (2019) 023.

[93] Y.S. Myung and D.-C. Zou, Int. J. Mod. Phys. D 28, 1950114 (2019).

[94] Y. Brihaye and B. Hartmann, J. High Energy Phys. 09 (2019) 049.

[95] O. J. Tattersall and P. G. Ferreira, Phys. Rev. D 99, 104082 (2019).

[96] N. Andreou, N. Franchini, G. Ventagli, and T. P. Sotiriou, Phys. Rev. D 99, 124022 (2019).

[97] Q. Liang, J. Sakstein, and M. Trodden, Phys. Rev. D 100, 063518 (2019).

[98] L. Hui, D. Kabat, X. Li, L. Santoni, and S. S. C. Wong, J. Cosmol. Astropart. Phys. 06 (2019) 038.

[99] D. Q. Tuan and S. H. Q. Nguyen, Commun. Phys. 29, 173 (2019).

[100] T. Do et al., Science 365, 664 (2019).

[101] P. G. S. Fernandes, C. A. R. Herdeiro, A. M. Pombo, E. Radu, and N. Sanchis-Gual, Phys. Rev. D 100, 084045 (2019).

[102] R. A. Konoplya and A. Zhidenko, Phys. Rev. D 100, 044015 (2019).

[103] N. Franchini and T. P. Sotiriou, arXiv:1903.05427.

[104] A. Hees, O. Minazzoli, E. Savalle, Y. V. Stadnik, P. Wolf, and B. Roberts, arXiv:1905.08524.

[105] T. Anson, E. Babichev, and S. Ramazanov, Phys. Rev. D 100, 104051 (2019).

[106] M. Khalil, N. Sennett, J. Steinhoff, and A. Buonanno, Phys. Rev. D 100, 124013 (2019).

[107] C. de Rham and J. Zhang, Phys. Rev. D 100, 124023 (2019).

[108] G. Aguilar-Perez, M. Cruz, S. Lepe, and I. Moran-Rivera, arXiv:1907.06168.

[109] R. A. Konoplya, T. Pappas, and A. Zhidenko, arXiv: 1907.10112.

[110] Y.-X. Gao and D.-J. Liu, arXiv:1908.01346.

[111] T. Ikeda, T. Nakamura, and M. Minamitsuji, Phys. Rev. D 100, 104014 (2019).

[112] F.-L. Julie and E. Berti, Phys. Rev. D 100, 104061 (2019).

[113] F. M. Ramazanoglu and K. I. Unluturk, Phys. Rev. D 100, 084026 (2019).

[114] K. V. Aelst, E. Gourgoulhon, P. Grandclement, and C. Charmousis, Classical Quantum Gravity 37, 035007 (2020).

[115] J. Barrientos, F. Cordonier-Tello, C. Corral, F. Izaurieta, P. Medina, E. Rodriguez, and O. Valdivia, Phys. Rev. D 100, 124039 (2019).

[116] C. Martinez, R. Troncoso, and J. Zanelli, Phys. Rev. D 67, 024008 (2003).
[117] T. J. T. Harper, P. A. Thomas, E. Winstanley, and P. M. Young, Phys. Rev. D 70, 064023 (2004).

[118] M. Henneaux, C. Martinez, R. Troncoso, and J. Zanelli, Phys. Rev. D 70, 044034 (2004); C. Martinez, R. Troncoso, and J. Zanelli, Phys. Rev. D 70, 084035 (2004); C. Erices and C. Martinez, Phys. Rev. D 97, 024034 (2018).

[119] E. Radu and E. Winstanley, Phys. Rev. D 72, 024017 (2005).

[120] A. Anabalon and H. Maeda, Phys. Rev. D 81, 041501 (2010).

[121] D. Hosler and E. Winstanley, Phys. Rev. D 80, 104010 (2009).

[122] C. Charmousis, T. Kolyvaris, and E. Papantonopoulos, Classical Quantum Gravity 26, 175012 (2009); T. Kolyvaris, G. Koutsoumbas, E. Papantonopoulos, and G. Siopsis, Gen. Relativ. Gravit. 43, 163 (2011).

[123] K. i. Maeda, N. Ohta, and Y. Sasagawa, Phys. Rev. D 83, 044051 (2011); Z. K. Guo, N. Ohta, and T. Torii, Prog. Theor. Phys. 121, 253 (2009); N. Ohta and T. Torii, Prog. Theor. Phys. 121, 959 (2009); 122, 1477 (2009).

[124] S. G. Saenz and C. Martinez, Phys. Rev. D 85, 104047 (2012).

[125] M. M. Caldarelli, C. Charmousis, and M. Hassaine, J. High Energy Phys. 10 (2013) 015.

[126] P. A. Gonzalez, E. Papantonopoulos, J. Saavedra, and Y. Vasquez, J. High Energy Phys. 12 (2013) 021.

[127] M. B. Gaete and M. Hassaine, Phys. Rev. D 88, 104011 (2013); J. High Energy Phys. 11 (2013) 177.

[128] G. Giribet, M. Leoni, J. Oliva, and S. Ray, Phys. Rev. D 89, 085040 (2014).

[129] J. B. Achour and H. Liu, Phys. Rev. D 99, 064042 (2019).

[130] Y. Brihaye, B. Hartmann, and J. Urrestilla, J. High Energy Phys. 06 (2018) 074; Y. Brihaye and B. Hartmann, J. High Energy Phys. 01 (2019) 142.

[131] A. Bakopoulos, G. Antoniou, and P. Kanti, Phys. Rev. D 99, 064003 (2019).

[132] J. B. Achour and H. Liu, Phys. Rev. D 99, 064042 (2019).

[133] P. Kanti, A. Bakopoulos, and N. Pappas, Proc. Sci., CORFU2018 (2019) 091.

[134] Y. Brihaye, C. Herdeiro, and E. Radu, arXiv:1910.05286.

[135] P. Kanti, B. Kleihaus, and J. Kunz, Phys. Rev. Lett. 107, 271101 (2011); Phys. Rev. D 85, 044007 (2012).

[136] G. Antoniou, A. Bakopoulos, P. Kanti, B. Kleihaus, and J. Kunz, Phys. Rev. D 101, 024033 (2020).

[137] B. Kleihaus, J. Kunz, and P. Kanti, arXiv:1910.02121.

[138] C. A. R. Herdeiro and J. M. S. Oliveira, Classical Quantum Gravity 36, 105015 (2019).

[139] V. I. Afonso, G. J. Olmo, E. Orazi, and D. Rubiera-Garcia, J. Cosmol. Astropart. Phys. 12 (2019) 044.

[140] C. A. R. Herdeiro, J. M. S. Oliveira, and E. Radu, Eur. Phys. J. C 80, 23 (2020).

[141] P. Canate, J. Sultana, and D. Kazanas, Phys. Rev. D 100, 064007 (2019).

[142] P. Canate and N. Breton, Phys. Rev. D 100, 064067 (2019).

[143] I. Antoniadis, J. Rizos, and K. Tamvakis, Nucl. Phys. B415, 497 (1994).

[144] J. Rizos and K. Tamvakis, Phys. Lett. B 326, 57 (1994).

[145] P. Kanti, J. Rizos, and K. Tamvakis, Phys. Rev. D 59, 083512 (1999).

[146] P. Kanti, R. Gannouji, and N. Dadhich, Phys. Rev. D 92 , 041302 (2015); 92, 083524 (2015). 